\title{
Selective role of Nck1 in atherogenic inflammation and plaque formation
}

\author{
Mabruka Alfaidi, ${ }^{1,2}$ Christina H. Acosta, ${ }^{3}$ Dongdong Wang, ${ }^{1,2}$ James G. Traylor, ${ }^{1,2}$ and A. Wayne Orr ${ }^{1,2,3,4}$ \\ 'Department of Pathology and Translational Pathobiology, ${ }^{2}$ Center for Cardiovascular Diseases and Sciences, ${ }^{3}$ Department of Cell Biology and Anatomy, and ${ }^{4}$ Department of Molecular and Cellular \\ Physiology, LSU Health Shreveport, Shreveport, Louisiana, USA.
}

\begin{abstract}
Although the Canakinumab Anti-Inflammatory Thrombosis Outcomes Study (CANTOS) established the role of treating inflammation in atherosclerosis, our understanding of endothelial activation at atherosclerosis-prone sites remains limited. Disturbed flow at atheroprone regions primes plaque inflammation by enhancing endothelial NF- $\mathrm{B}$ signaling. Herein, we demonstrate a role for the Nck adaptor proteins in disturbed flow-induced endothelial activation. Although highly similar, only Nck1 deletion, but not Nck2 deletion, limited flow-induced NF-кB activation and proinflammatory gene expression. Nck1-knockout mice showed reduced endothelial activation and inflammation in both models, disturbed flow- and high fat diet-induced atherosclerosis, whereas Nck2 deletion did not. Bone marrow chimeras confirmed that vascular Nck1, but not hematopoietic Nck1, mediated this effect. Domain-swap experiments and point mutations identified the Nck1 SH2 domain and the first SH3 domain as critical for flow-induced endothelial activation. We further characterized Nck1's proinflammatory role by identifying interleukin 1 type I receptor kinase-1 (IRAK-1) as a Nck1-selective binding partner, demonstrating that IRAK1 activation by disturbed flow required Nck1 in vitro and in vivo, showing endothelial Nck1 and IRAK-1 staining in early human atherosclerosis, and demonstrating that disturbed flow-induced endothelial activation required IRAK-1. Taken together, our data reveal a hitherto unknown link between Nck1 and IRAK-1 in atherogenic inflammation.
\end{abstract}

\section{Introduction}

Atherosclerosis, a chronic lipid-driven arterial inflammatory disease (1), develops at sites of local endothelial activation, a proinflammatory shift in endothelial cell phenotype (2). Local hemodynamic shear stress, a frictional force of blood flow on the endothelium (3), confers protection or susceptibility to endothelial activation, with atheroprotective laminar flow limiting endothelial activation and atheroprone disturbed flow stimulating endothelial activation, characterized by cytoskeletal remodeling, endothelial stiffening, and nuclear factor- $\mathrm{kB}$-driven (NF-kBdriven) proinflammatory gene expression (3-5). Activated endothelial cells recruit monocytes from the circulation, and in the context of hypercholesterolemia, these monocytes accumulate lipid to drive early fatty streak formation (6). Recruitment of smooth muscle cells from the underlying media contribute to atheroma formation and drive the production of a collagen-rich protective fibrous cap that limits plaque vulnerability to rupture (7). Although recent results from the Canakinumab AntiInflammatory Thrombosis Outcomes Study (CANTOS) highlight the important role of limiting inflammation in the treatment of atherosclerosis (8), our understanding of the mechanisms regulating flow-induced endothelial activation remain limited.

\section{Related Commentary: p. 3968}

Conflict of interest: The authors have declared that no conflict of interest exists. Copyright: () 2020, American Society for Clinical Investigation.

Submitted: February 18, 2020; Accepted: May 13, 2020; Published: July 13, 2020

Reference information: J Clin Invest. 2020;130(8):4331-4347.

https://doi.org/10.1172/JCl135552.
The Nck family of adaptor proteins (Nck1 and Nck2) are ubiquitously expressed and share approximately $68 \%$ amino acid identity (9). Nck adaptor proteins lack enzymatic activity but control the formation of signaling complexes through 3 tandem Src homology 3 (SH3) domains and 1 C-terminal SH2 domain (10). SH2 domains bind with high affinity to tyrosine-phosphorylated proteins, whereas the $\mathrm{SH} 3$ domains bind to proline-rich sequences (PXXP) in signaling partners, suggesting that Nck serves to couple tyrosine kinase signaling to the activation of downstream pathways (11). The 2 highly similar Nck proteins are expressed by different genes (11) that play redundant roles during development, as deletion of both Nck isoforms results in an embryonic lethal phenotype due to impaired vasculogenesis, while deletion of only one isoform does not (9). Although Nck1 and Nck2 play redundant roles in regulating angiogenesis in mouse models of retinopathy (12), Nck2 may play a dominant role in PDGF-induced actin polymerization in NIH3T3 cells (13). In contrast, Nck1 plays a more dominant role in $\mathrm{T}$ cell receptor-induced ERK activation (14), suggesting noncompensating roles during phenotypic regulation after development. However, the signaling effects of Nck1/2 outside the context of cytoskeletal remodeling are incompletely understood.

We have previously demonstrated that treatment with a membrane-permeant peptide corresponding to a Nck-binding PXXP sequence blunts flow-induced endothelial activation in vitro and significantly reduces both inflammation and vascular permeability at atheroprone areas exposed to disturbed flow in vivo (15). However, this peptide could be reducing atherosclerotic inflammation through off-target effects on other SH3-containing adaptor proteins. Furthermore, the antiinflammatory effects observed 
A

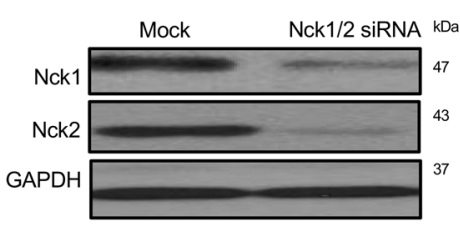

B

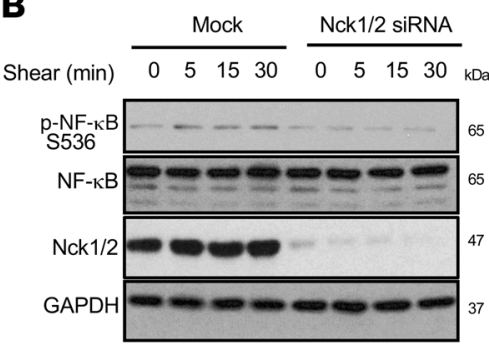

C

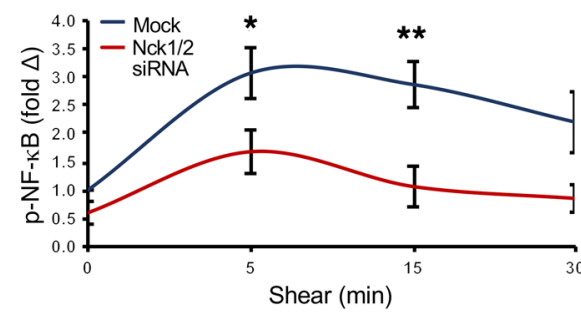

E

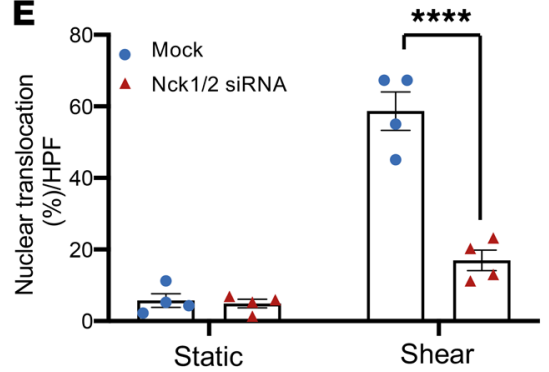

D

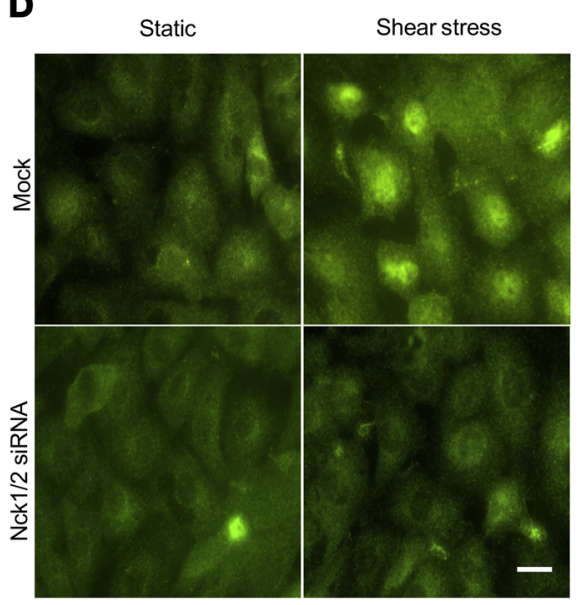

$\mathbf{F}$

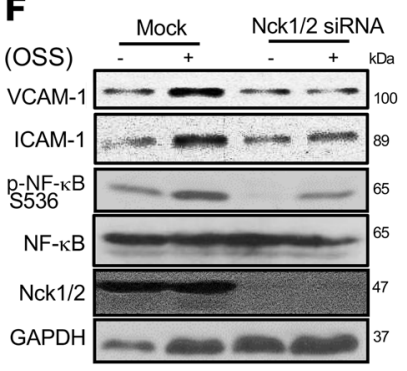

G

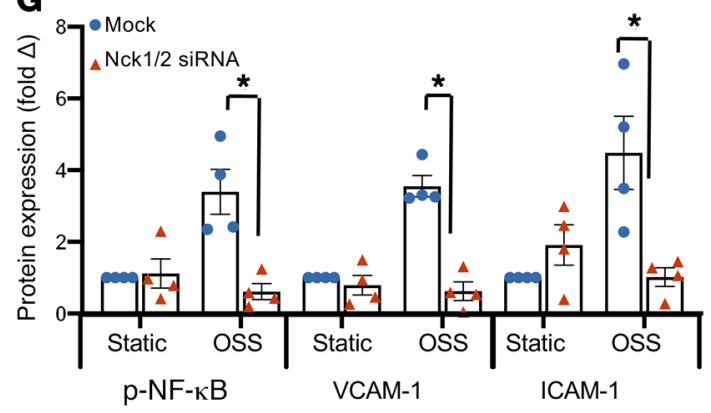

H

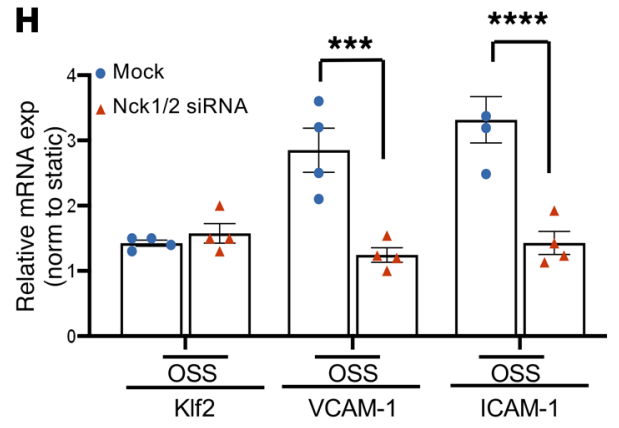

Figure 1. Nck1/2 deletion ameliorates shear stress-induced NF-кB activation. (A) Human aortic endothelial cells (HAECs) were transfected with siRNA specific for Nck1/2, and transfection efficiency was assessed using Western blotting. (B and C) HAECs were subjected to acute shear stress for the indicated times, and NF- $\mathrm{kB}$ activation was assessed by detection of $\mathrm{p} 65$ serine 536 phosphorylation using Western blotting. Densitometric analysis was performed using Image). (D and E) p65 nuclear translocation was measured after 45 minutes of shear stress in Nck1/2 siRNA-treated and mock control cells. Images were analyzed using NIS Elements software. Scale bar: $50 \mu \mathrm{m}$. Data are presented as the mean $\pm \mathrm{SEM}, n=4 .{ }^{*} P<0.05,{ }^{* *} P<0.01,{ }^{* * * *} P<0.0001$ by 2-way ANOVA followed by Bonferroni's post hoc test. HPF, high-power field. (F and $\mathbf{G}$ ) HAECs were transfected with Nck1/2 siRNA, and oscillatory shear stress-induced (OSS-induced; \pm 5 dynes $/ \mathrm{cm}^{2}$ with 1 dyne $/ \mathrm{cm}^{2}$ forward flow) proinflammatory gene expression (VCAM-1, ICAM-1) and proinflammatory signaling ( $\mathrm{p}-\mathrm{NF}-\mathrm{KB}$ Ser536) were assessed by Western blotting. $n=4$ in $\mathbf{G}$. ${ }^{*} P<0.05$ by Kruskal-Wallis test. (H) HAECs were treated as in F, and mRNA expression was assessed by qRT-PCR $(n=4) .{ }^{* *} P<0.001$, ${ }^{* * *} P<0.0001$ by 2 -way ANOVA followed by Bonferroni's post hoc test.

in vivo could be due to effects of this peptide on other cell types besides endothelial cells (16). Interestingly, the Nck1 gene locus at chromosome $3 \mathrm{q} 22.3$ has been associated with atherosclerosis susceptibility and myocardial infarction (17), and a genome-wide association study identified Nck1 as a novel coronary artery disease susceptibility locus (18). Herein, we used both cell culture and animal models of disturbed blood flow and high fat diet-induced atherosclerosis to characterize the noncompensating functions of Nck1 and Nck2 in disturbed flow-induced endothelial activation.

\section{Results}

$N c k 1 / 2$ deletion and its effects on shear stress-induced endothelial activation. Using the EndoDB database of endothelial transcriptomics data (19), we found that Nck1 and Nck2 show similar expression across a variety of human and mouse endothelial cell studies. To investigate the direct roles of Nck1/2 in shear stress-induced endothelial activation, we adopted a loss-of-function model in which the cellular levels of Nck1 and Nck2 were reduced by siRNA transfection. The efficiency of transfection was confirmed using Western blotting, with a 70\% knockdown of Nck1 and 84\% knockdown of Nck2 (Figure 1A). Endothelial cells subjected to acute laminar shear stress show an early upregulation of proinflammatory signaling, driven in part by the same signaling pathways that mediate cytoskeletal alignment (20). As such, these signals are diminished as the cells adapt to laminar flow but are sustained in disturbed flow due to the inability of the cells to adapt. Because the acute endothelial response to shear stress models the sustained signaling observed with chronic disturbed flow (20), endothelial cells were subjected to acute shear stress for $0,5,15$, or 30 minutes, and the early signaling effects were measured. NF- $\mathrm{kB}$ activation was assessed by measuring $\mathrm{p} 65 \mathrm{~S} 536$ phosphorylation or nuclear translocation. Nck1/2-knockdown cells showed a significant reduction in both NF-KB phosphorylation (Figure 1, $\mathrm{B}$ and $\mathrm{C})$ and NF- $\mathrm{\kappa B}$ nuclear translocation $(55 \% \pm 7.8 \%$ mock vs. $13 \% \pm 1.4 \%$ in Nck1/2 siRNA, $P<0.0001$ ) (Figure $1, \mathrm{D}$ and E). To confirm the combined effect of Nck1/2 knockdown, we used 
A

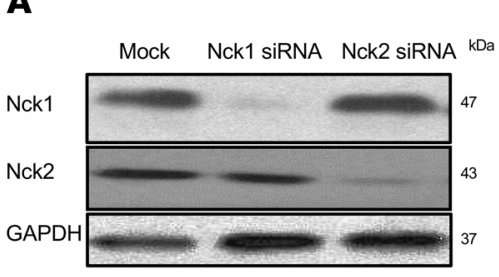

C

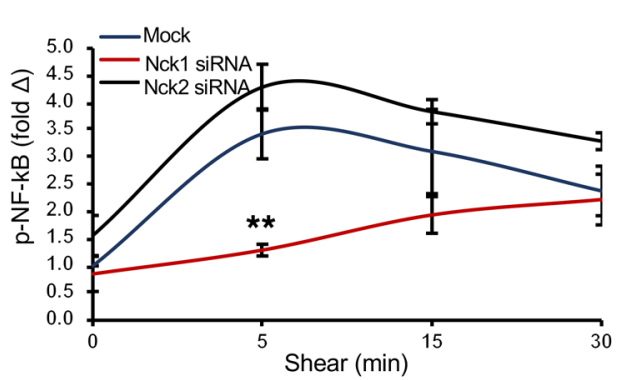

G

$\mathbf{F}$

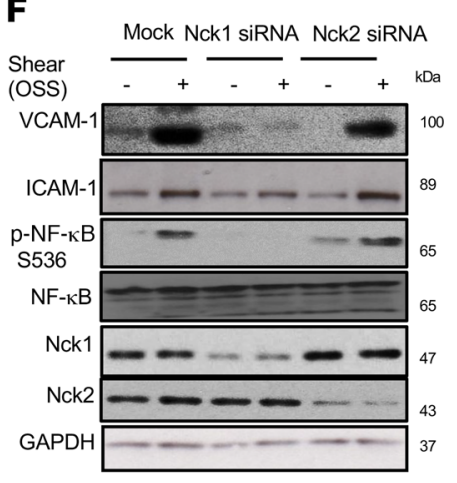

B

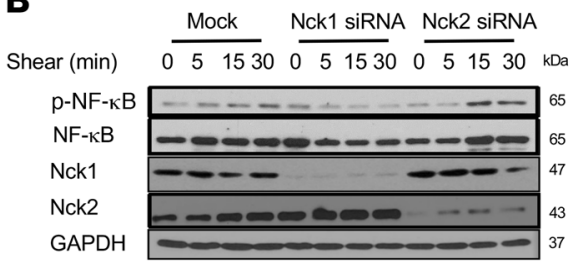

E

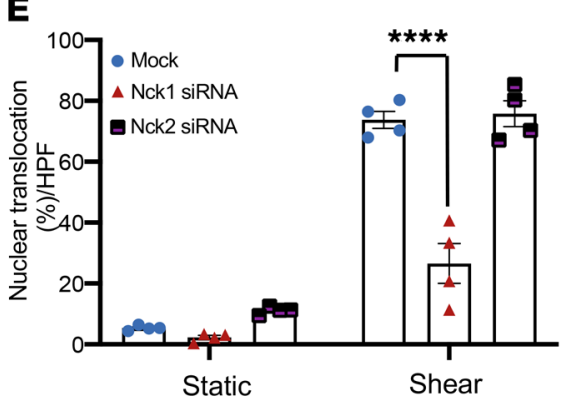

D

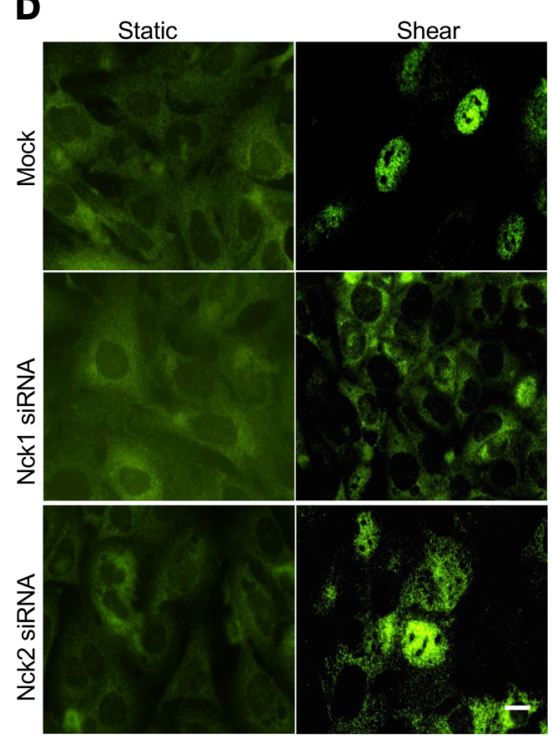

H

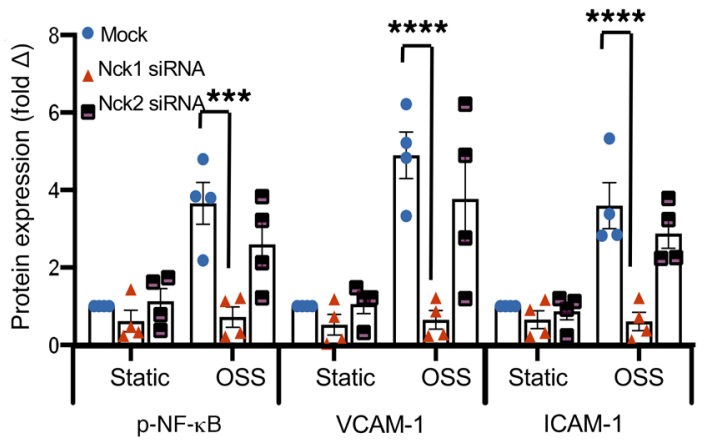

Figure 2. Nck1, but not Nck2, deletion ameliorates shear stress-induced activation. (A) Transfection efficiency of selective Nck1 and Nck2 knockdown in HAECs using siRNA. (B-E) HAECs lacking either Nck1 or Nck2 were subjected to acute shear stress for the indicated times, and NF- $k B$ activation was assessed by measuring (B and $\mathbf{C}$ ) NF-KB phosphorylation and ( $\mathbf{D}$ and $\mathbf{E})$ nuclear translocation. Scale bar: $50 \mu \mathrm{m}$. Data are the mean \pm SEM, $n=4$. ( $\mathbf{F}$ and $\mathbf{G})$ HAECs were transfected with either Nck1 or Nck2 siRNA, and oscillatory shear stress-induced (OSS-induced, 18 hours) proinflammatory gene expression (ICAM-1/VCAM-1) and signaling ( $\mathrm{p}-\mathrm{NF}-\kappa \mathrm{B}$ Ser536) were assessed by Western blotting. (H) Cells transfected as in $\mathbf{F}$ and mRNA expression of KIf2, VCAM-1, and ICAM- 1 were measured using qRT-PCR. Data are from $n=4$ and presented as the mean \pm SEM. ${ }^{*} P<0.05,{ }^{* *} P<0.01,{ }^{* * *} P<0.001,{ }^{* * * *} P<0.0001$ by 2-way ANOVA followed by Bonferroni's post hoc test.

CRISPR/Cas9 editing to generate a stable human aortic endothelial cell (HAEC) line lacking in both Nck1 and Nck2. Nck1/2 double-knockout (Nck1/2-DKO) cells show a similar decrease in shear stress-induced NF- $\mathrm{BB}$ phosphorylation (Supplemental Figure 1, A and B; supplemental material available online with this article; https://doi.org/10.1172/JCI135552DS1) and nuclear translocation (Supplemental Figure 1, C and D) compared with scrambled controls (Supplemental Figure 1, A-D). Similar results were also observed in mouse aortic endothelial cells (MAECs) isolated from endothelial cell-specific Nck1/2-DKO mice (iEC-Nck1/2 DKO; VE-cadherinCreERT2 ${ }^{\mathrm{tg} / ?}, \mathrm{Nck}^{-/-}, \mathrm{Nck}^{\mathrm{fl} / \mathrm{fl}}, \mathrm{ApoE}^{-/-}$) (Supplemental Figure 1, E-G).

In response to chronic oscillatory shear stress (OSS), NF- $\kappa \mathrm{B}$ activation drives proinflammatory gene expression, including ICAM-1 and VCAM-1 (21). We found that siRNA-mediated Nck1/2 depletion reduced OSS-induced VCAM-1 and ICAM-1 protein expression (Figure 1, F and G). Compared with the mock controls, Nck1/2 siRNA-treated cells showed significantly less NF- $\mathrm{B}$ activation (Figure 1, F and $\mathrm{G}$ ) and mRNA expression of ICAM-1 and VCAM-1 (Figure 1H). Shear stress-induced expression of the atheroprotective gene Klf2 was not affected by Nck1/2 depletion (Figure 1H). Consistent with these data, Nck1/2-DKO endothelial cells show a similar reduction in OSS-induced NF- $\kappa$ B activation (Supplemental Figure 1, H and I) and VCAM-1/ICAM-1 expression (Supplemental Figure 1, H-J). Taken together, these data suggest that Nck1/2 expression is required to couple atheroprone hemodynamics to NF- $\kappa \mathrm{B}$ activation and proinflammatory gene expression.

Deletion of Nck1, but Not Nck2, ameliorates shear stress-induced endothelial activation. Even though they are expressed by different genes, Nck1 and Nck2 proteins share a high sequence identity (68\% overall) (10) and their functions are generally regarded as overlapping (22). However, emerging evidence has suggested the independent contribution of the 2 isoforms in a variety of responses, including $\mathrm{T}$ cell activation, cytokinesis, and podocyte cytoskeletal dynamics (23-25). To investigate the selective roles of Nck1 and Nck2, we used Nck1- and Nck2-selective siRNAs that result 
A

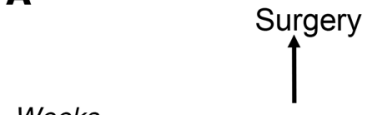

\begin{tabular}{crcrr} 
Weeks 8 & 10 & 12 & $48 \mathrm{~h}$ & 13 \\
$\begin{array}{c}\text { Tamoxifen } \\
\text { injections }\end{array}$ & & Normal chow diet & \\
\hline & & $\uparrow$ & $\uparrow$ \\
& & mRNA & Histology
\end{tabular}

B

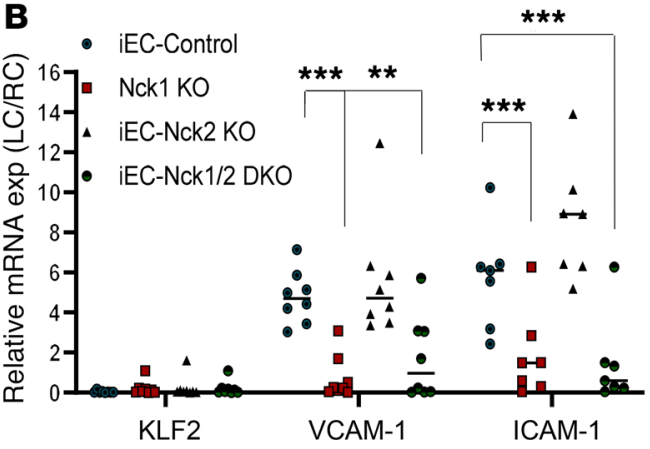

D

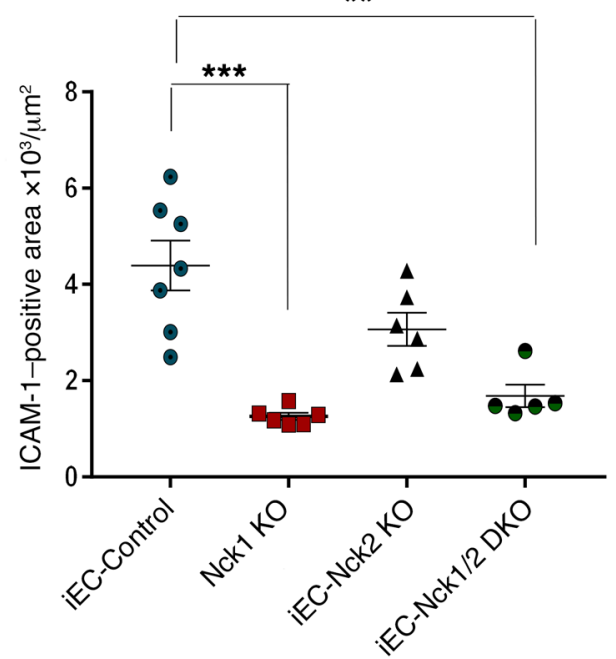

$\mathbf{F}$

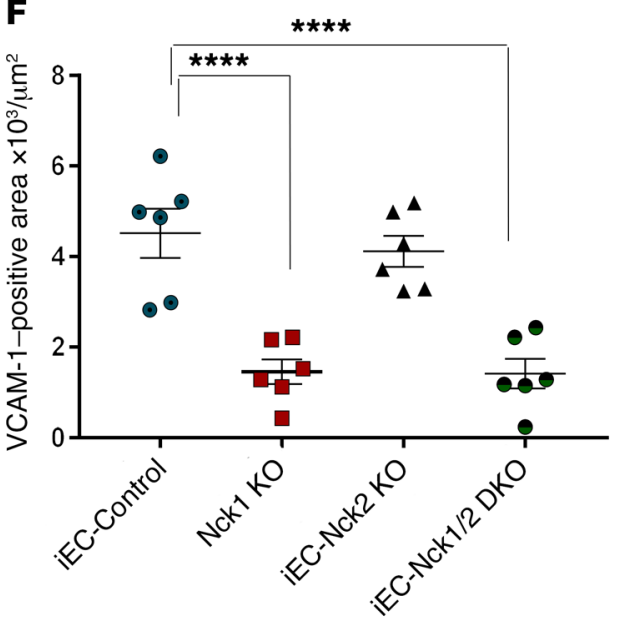

C

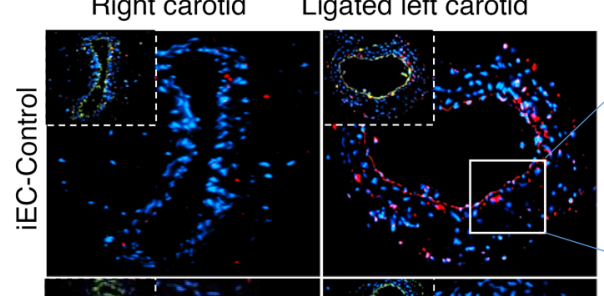

Right carotid

Ligated left carotid

$\frac{0}{\frac{1}{0}}$
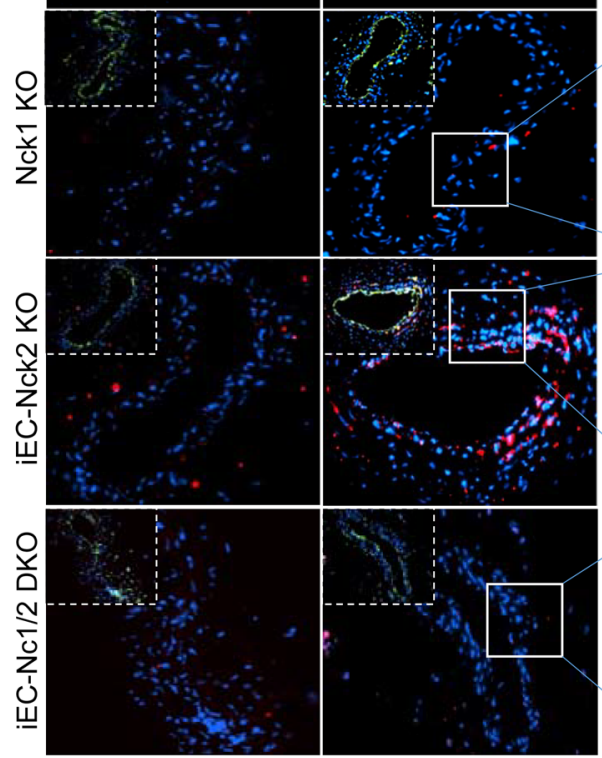

E

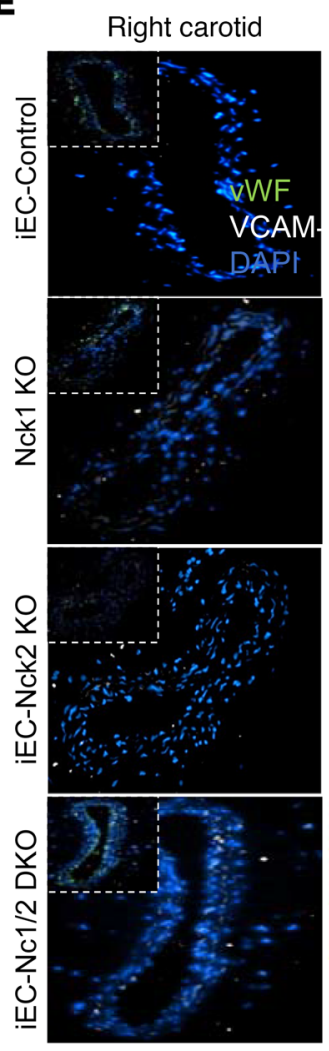

ICAM-1

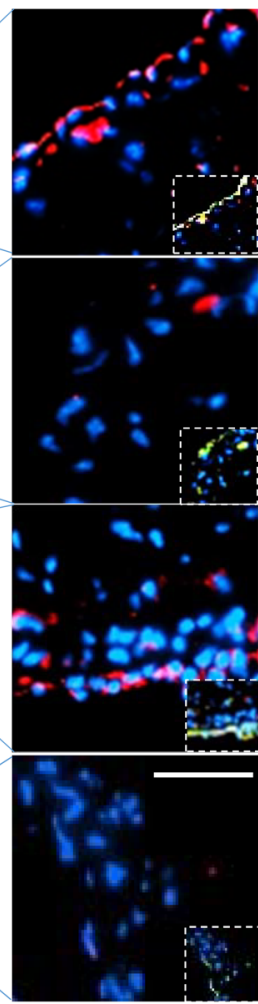

VCAM-1

Ligated left carotid
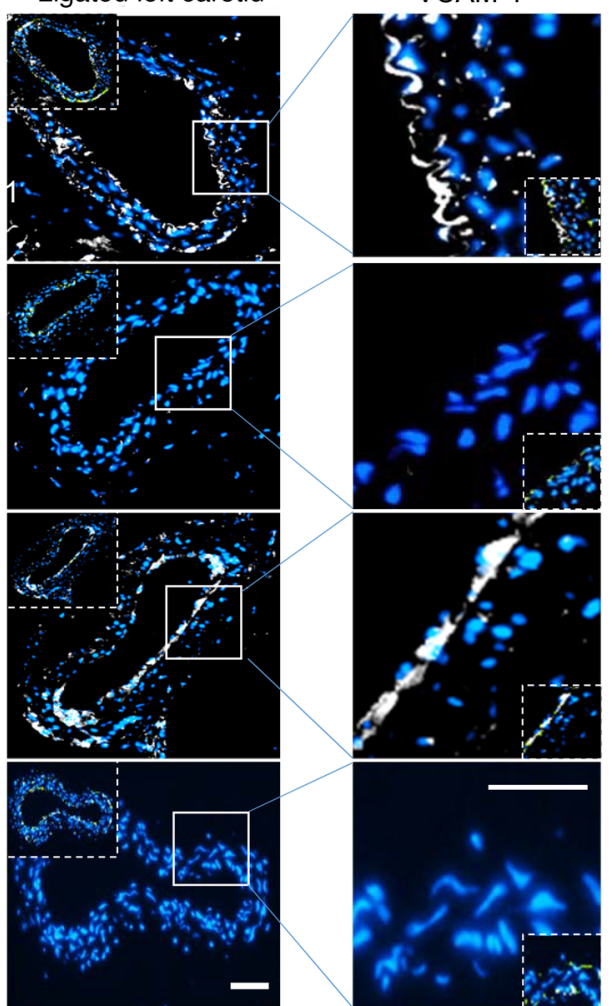
Figure 3. Ablation of Nck1, but not Nck2, blunts partial carotid ligationinduced inflammation. (A) Schematic of the study in which 4 groups of mice were subjected to the ligation surgery at the indicated time. (B) Endothelial mRNA analysis from iEC-Control, Nck1-KO, iEC-Nck2-KO, and iEC-Nck1/2-DKO mice. mRNA from the ligated left carotid (LC) was normalized to the unligated right carotid (RC) and to the housekeeping gene $\beta$-microglobulin. ${ }^{* *} P<0.01$; ${ }^{* *} P<0.001$ by 2 -way ANOVA followed by Bonferroni's post hoc test. (C-F) ICAM-1 (red) and VCAM-1 (white) in the ligated left carotid compared with the unligated right carotid arteries among experimental groups. Endothelial cells were stained for von Willebrand factor (VWF in the dashed boxes) and the nuclei counterstained with DAPI. Scale bars: $200 \mu \mathrm{m}$ (left 2 columns in $\mathbf{C}$ and $\mathbf{E}$ ) and $50 \mu \mathrm{m}$ (right columns). Images analyzed using NIS Elements software, from $n=7-10$ mice/group. Data are the mean $\pm \mathrm{SEM}$. ${ }^{* *} P<0.01$; ${ }^{* *} P<0.001$; ${ }^{* * *} P<$ 0.0001 by 1 -way ANOVA followed by Tukey's post hoc test.

in a $75 \%$ and $85 \%$ knockdown, respectively, without affecting the expression of the other isoform (Figure 2A). In response to shear stress, Nck1-depleted cells showed significantly less NF-кB p65 phosphorylation (Figure 2, B and $\mathrm{C}$ ) and nuclear translocation (Figure 2, D and E), whereas Nck2 depletion did not affect NF- $\kappa$ B activation by flow. To confirm these effects, we used lentiviral shRNA constructs to selectively deplete Nck1 (100\% knockdown) and Nck2 (90\% knockdown) (Supplemental Figure 2A). Similar to the siRNA data, only HAECs expressing Nck1 shRNA showed significant amelioration of NF- $\mathrm{KB}$ phosphorylation (Supplemental Figure 2, B and C) and nuclear translocation (Supplemental Figure 2, D and E), whereas cells expressing Nck2 shRNA did not significantly differ from cells expressing scrambled shRNA. MAECs isolated from Nck1-KO mice showed similar results, with remarkable reduction in NF- $\mathrm{BB}$ activation following shear stress, whereas MAECs from iEC-Nck2-KO mice showed the usual shear stress-induced NF- $\kappa$ B activation (Supplemental Figure 2, F-H). To assess the specific role of Nck1 in atheroprone disturbed flow models, HAECs transfected with Nck1 and Nck2 siRNA or shRNA were exposed to OSS for 18 hours, and proinflammatory signaling $(\mathrm{NF}-\kappa \mathrm{B})$ and proinflammatory gene expression (VCAM-1/ICAM1) were assessed. Oscillatory flow-induced NF- $\kappa B$ activation (p65 Ser536 phosphorylation) and VCAM-1/ICAM-1 protein expression (Figure 2, F and $\mathrm{G}$ ) and mRNA levels (Figure 2H) were blunted by Nck1 siRNA (Figure 2, F-H) and Nck1 shRNA (Supplemental Figure 2, I-K), whereas Nck2 depletion (siRNA and shRNA) had no significant effects on any of these responses.

Although these data identify Nck1 as a critical regulator of $\mathrm{NF}-\kappa \mathrm{B}$ activation and proinflammatory gene expression by atheroprone hemodynamics, Nck1 did not affect all shear stress responses. Neither Nck1 nor Nck2 depletion affected Klf2 expression under OSS (Figure $2 \mathrm{H}$ and Supplemental Figure $2 \mathrm{~K}$ ) or activation of other classic shear stress-induced signaling pathways, such as Akt, eNOS, and ERK1/2 phosphorylation (Supplemental Figure 3). Collectively, these data demonstrate a critical role for Nck1 in endothelial activation by atheroprone flow, whereas Nck2 has no effect.

Nck1 mediates disturbed flow-induced endothelial activation in vivo. Having shown that Nck1 regulates endothelial activation by atheroprone flow in vitro, we sought to investigate the differential effects of Nck1 and Nck2 in an in vivo model of disturbed flow. Following tamoxifen induction, inducible endothelial cell-specific control mice (iEC-Control; VE-cadherinCreERT2 ${ }^{\mathrm{tg} / \text { ? }}, \mathrm{ApoE}^{-/-}$), Nck1-KO (VE-cadherinCreERT2 ${ }^{\mathrm{t} /} /$ ? $\mathrm{Nck1}^{-/}, \mathrm{ApoE}^{-/-}$), endothelial cell-specific Nck2-KO (iEC-Nck2-KO; VE-cadherinCreERT2to/?, $\left.\mathrm{Nck}^{\mathrm{f} / \mathrm{fl}}, \mathrm{ApoE}^{-/-}\right)$, and endothelial cell-specific Nck1/2-DKO mice (iEC-Nck1/2 DKO; VE-cadherinCreERT2 ${ }^{\mathrm{tg} / \text { ? }}, \mathrm{Nck} 1^{-/}, \quad \mathrm{Nck} 2^{\mathrm{f} / \mathrm{ll}}$, $\mathrm{ApoE}^{-/-}$) were subjected to partial carotid ligation (PCL) to induce disturbed flow-associated endothelial activation specifically in the left carotid artery $(26,27)$. Changes in endothelial mRNA expression were assessed after 48 hours, whereas changes in inflammatory gene expression and macrophage recruitment were assessed after 7 days (Figure 3A). To assess endothelial activation, endothelial mRNA was isolated from the left and right carotid vessels by TRIzol flush after tissue harvesting (28). The purity of intimal mRNA and medial/adventitial mRNA was confirmed by measuring platelet endothelial cell adhesion molecule-1 (PECAM-1) and $\alpha$-smooth muscle actin (SMA) expression (Supplemental Figure 4, A and B). Furthermore, endothelial cell-specific deletion of Nck2 was confirmed in iEC-Nck2-KO and iEC-DKO mice, as Nck2 mRNA expression was depleted in the intimal but not the medial/adventitial fractions (Supplemental Figure 4, C and D). Klf2 showed decreased expression in the ligated left carotid compared with the right carotid control, confirming an OSS flow-associated gene expression profile (Figure 3B). However, this downregulation did not differ among experimental animals. Nck1 knockouts showed a pronounced reduction in oscillatory flow-induced VCAM-1 and ICAM-1 mRNA expression (Figure 3B). However, Nck2 deletion (iEC-Nck2-KO) did not affect disturbed flow-induced VCAM-1 and ICAM-1 expression, and VCAM-1/ ICAM-1 mRNA expression did not significantly decrease in iECNck1/2-DKO mice compared with Nck1-KO mice (Figure 3B).

To examine early atherogenic remodeling in the ligated carotid arteries, tissues were collected 7 days after ligation and assessed for markers of inflammation by immunohistochemistry. Consistent with early changes in mRNA expression, VCAM-1 and ICAM-1 protein levels were significantly reduced in Nck1-KO mice after PCL compared with iEC-Control mice (Figure 3, C-F), whereas iEC-Nck2-KO animals were similar to controls. Similarly, Nck1$\mathrm{KO}$ mice showed a significant reduction in intimal macrophage recruitment (Mac-2 $2^{+}$area) compared with iEC-Control mice (Figure 4, A and B). Nck1-KO mice also showed significantly reduced adventitial macrophage content (Figure 4, A and C), but endothelial Nck2 deletion (iEC-Control vs. iEC-Nck2-KO; Nck1-KO vs. iEC-Nck1/2-DKO) did not affect intimal or adventitial macrophage infiltration (Figure $4, \mathrm{~A}-\mathrm{C}$ ). Specificity of the Mac-2 staining was verified using isotype-control antibodies (Supplemental Figure 5). Taken together, our data suggest a direct role for Nck1 in regulating endothelial activation and macrophage recruitment under atheroprone hemodynamics.

Nck1 deletion reduces atherosclerotic plaque formation. Atheroprone flow establishes local susceptibility to endothelial activation and to diet-induced atherosclerotic plaque development (29). Having observed differential effects for Nck1 and Nck2 in response to atheroprone flow-induced endothelial activation, we sought to determine if atherosclerotic plaque development was altered in Nck1-KO mice. iEC-Control, Nck1-KO, iEC-Nck2-KO, or iEC-DKO mice were fed high-fat diet (HFD) for 12 weeks to induce spontaneous atherosclerosis. No significant differences 
were observed in body weight over the 12 weeks of HFD feeding, though Nck1-KO mice tended to be smaller (Supplemental Figure $6 \mathrm{~A}$ ), and no changes were noted for heart weight (Supplemental Figure 6B) or for plasma cholesterol, triglycerides, or HDL levels among the experimental groups (Supplemental Figure 6, C-E). However, Nck1-KO mice showed significant reductions in the plasma levels of several proinflammatory mediators, including interleukin $1 \alpha$ (IL- $1 \alpha$ ), IL-1 $\beta$, TNF- $\alpha$, and MCP-1 (Supplemental Figure 7), highlighting the proinflammatory role of Nck1. Atherosclerotic lesion formation was assessed in 4 different vascular sites, including the aorta, the aortic roots, the brachiocephalic artery, and the right and left carotid sinuses. En face analysis of atherosclerosis in the aorta was assessed by Oil red $\mathrm{O}$ staining and calculated as the percentage lesion area compared with the total surface area of the aorta (Figure 5A). Although iEC-Nck2-KO mice did not differ from iEC-Controls, Nck1-KO mice show a significant reduction in plaque burden in the aorta (Figure 5, A and B), the carotid sinus (Figure 5, C and D), and brachiocephalic artery (Figure 5, $\mathrm{H}$ and I). Atherosclerosis did not decrease further in the iEC-Nck1/2-DKO compared with the Nck1-KO mice, suggesting that Nck1 is the key regulator and the endothelial Nck2 does not significantly contribute to atherogenic endothelial activation.

To assess atherosclerotic plaque characteristics in this model, plaques were stained for macrophage $\left(\mathrm{Mac}-2^{+}\right.$area) and smooth muscle $\left(\mathrm{SMA}^{+}\right)$levels. Compared with iEC-Control mice, Nck1-KO mice show a significant reduction in macrophage area in both the carotid sinus (Figure 5, E and F) and brachiocephalic arteries (Figure 5, J and K). Similarly, Nck1 deletion reduced the plaque smooth muscle $\left(\mathrm{SMA}^{+}\right)$area (Figure $5, \mathrm{G}$ and $\mathrm{L}$ ) and lipid core area (Supplemental Figure 8A) at these sites, consistent with the very early stages of plaque formation observed in Nck1-KO and iECNck1/2-DKO mice. Atherosclerotic lesions in the brachiocephalic artery and carotid sinuses tend to be less well developed than the plaques in the aortic root, potentially due to delayed onset of plaque formation at these sites (30). Unlike other sites, we did not observe any differences in plaque size in the aortic root (Supplemental Figure 8B). However, the plaques that formed showed reduced macrophage area (Supplemental Figure 8, C and D) and enhanced smooth muscle area (Supplemental Figure 8, C and E), suggestive of enhanced plaque stability.

Deletion of Nck1 confers atheroprotection in resident vascular bed cells compared with myeloid lineage cells. To determine the relative contribution of Nck1 deletion in either vascular cells or myeloid cells to atherosclerosis formation, we performed bone marrow transplantation experiments where Nck1-KO or Nck1-WT bone marrow was transplanted into irradiated Nck1-KO or Nck1-WT recipients. Successful chimeras were confirmed with the alteration in Nck1 expression in the myeloid lineage cells (Supplemental Figure 9, A and B). After 12 weeks of HFD, there were no significant changes in body weights (Supplemental Figure 10A) or plasma cholesterol levels (Supplemental Figure 10B) in mice lacking Nck1 expression in myeloid cells compared with other experimental groups. Consistent with our in vitro observations on endothelial activation, Nck1-KO mice still showed significant reduction in aortic atherosclerosis when reconstituted with Nck1-WT bone marrow, whereas Nck1-WT mice receiving bone marrow from Nck1-KO mice did not show any significant difference compared with Nck1-WT mice receiving Nck1-WT bone marrow (Figure 6, A and B). Similarly, carotid (Figure 6, C and D) and brachiocephalic atherosclerotic (Figure 6, H and I) lesions were 2- to 3-fold less in Nck1-KO mice receiving Nck1-WT bone marrow compared with controls $(P<0.0001, P<0.05$, respectively). Cross-sectional analysis of Mac- $2^{+}$and $\mathrm{SMA}^{+}$areas within carotid (Figure $6, \mathrm{E}^{-} \mathrm{G}$ ) and brachiocephalic artery (Figure 6, J-L) lesions showed significant reductions in carotid $\mathrm{Mac}^{-} 2^{+}$and $\mathrm{SMA}^{+}$areas only when $\mathrm{Nck} 1$ was depleted in the resident vascular cells but not when Nck1 was depleted in the bone marrow. These data support the role of vascular wall Nck1 in atherogenic inflammation and atherosclerotic plaque development and suggest that myeloid Nck1 expression does not contribute to atherosclerotic plaque formation.

Nck1 exerts the nonredundant effects on endothelial activation via its SH2 domain. To mechanistically understand why Nck1 but not the highly homologous Nck2 regulates endothelial activation, we conducted domain-swap experiments mixing the Nck1 SH2 domain with the Nck2 SH3 domains and the Nck2 SH2 domain with the Nck1 SH3 domains (Figure 7A). We confirmed similar expression levels of transfected constructs encoding Nck1, Nck2, the Nck1 SH2/Nck2 SH3 chimera, and the Nck2 SH2/Nck1 SH3 chimera in Nck1/2-DKO cells by Western blotting (Figure 7B). Nck1, but not Nck2, reexpression restored OSS-induced NF- $\mathrm{B}$ activation (Figure 7C), VCAM-1 expression (Figure 7D), and ICAM-1 expression (Supplemental Figure 11) in the Nck1/2-DKO cells. However, only the Nck1 SH2/Nck2 SH3 chimera showed a similar restoration, suggesting that the Nck1 SH2 domain is essential to form the signaling complex required for oscillatory flow-induced endothelial activation. However, the redundancy of the Nck1 and Nck2 SH3 domains does not exclude them as important for the activation of this response. To gain further insight into the Nck1 domains regulating oscillatory flow-induced $\mathrm{NF}-\kappa \mathrm{B}$ activation, we introduced single point mutations (Figure 7, $\mathrm{E}$ and F) to inactivate the Nck1 SH2 domain (R308M, Nck1 SH2*), the first SH3 domain (W38K, Nck1 SH3.1*), the second SH3 domain (W143K, Nck1 SH3.2*), or the third SH3 domain (W229K, Nck1 SH3.3*) (31). Following OSS exposure, NF-кB activation and VCAM-1 expression were only blunted in cells expressing the Nck1 SH2* and the Nck1 SH3.1* constructs (Figure 7, G-I), suggesting critical roles for Nck1 SH2-based phosphotyrosine binding and Nck1 SH3.1-based binding partners.

Nck1 interacts with IL-1 type I receptor kinase-1 in response to atheroprone hemodynamics. While these data suggest a critical role for Nck1 in atherogenic endothelial activation, the Nck1 binding partner involved remains unknown. A proteomics study by Jacquet et al. identified IL-1 type I receptor kinase-1 (IRAK-1) as a Nck1specific interacting partner (25). IRAK-1, a serine/threonine kinase extensively investigated in the IL-1 signaling pathway, is required for IL-1-induced NF- $\kappa B$ activation (32). To test the hypothesis that IRAK-1 phosphorylation requires Nck1, IRAK-1/Nck1 interactions were assessed by coimmunoprecipitation in endothelial cells exposed to flow. Following shear stress exposure, Nck1 interactions with IRAK- 1 were significantly enhanced (Figure 8, A-C), whereas Nck2 did not interact with IRAK-1 (Figure 8A), confirming the differences between Nck1 and Nck2 in shear stress signaling. Moreover, we exposed human endothelial cells to either atheroprotective laminar flow or atheroprone OSS, and assessed 
A

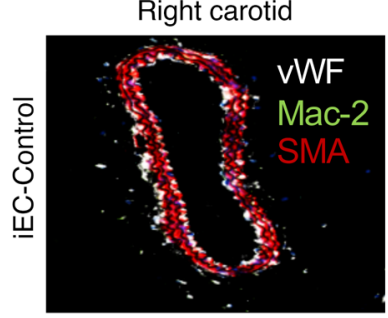

Ligated left carotid
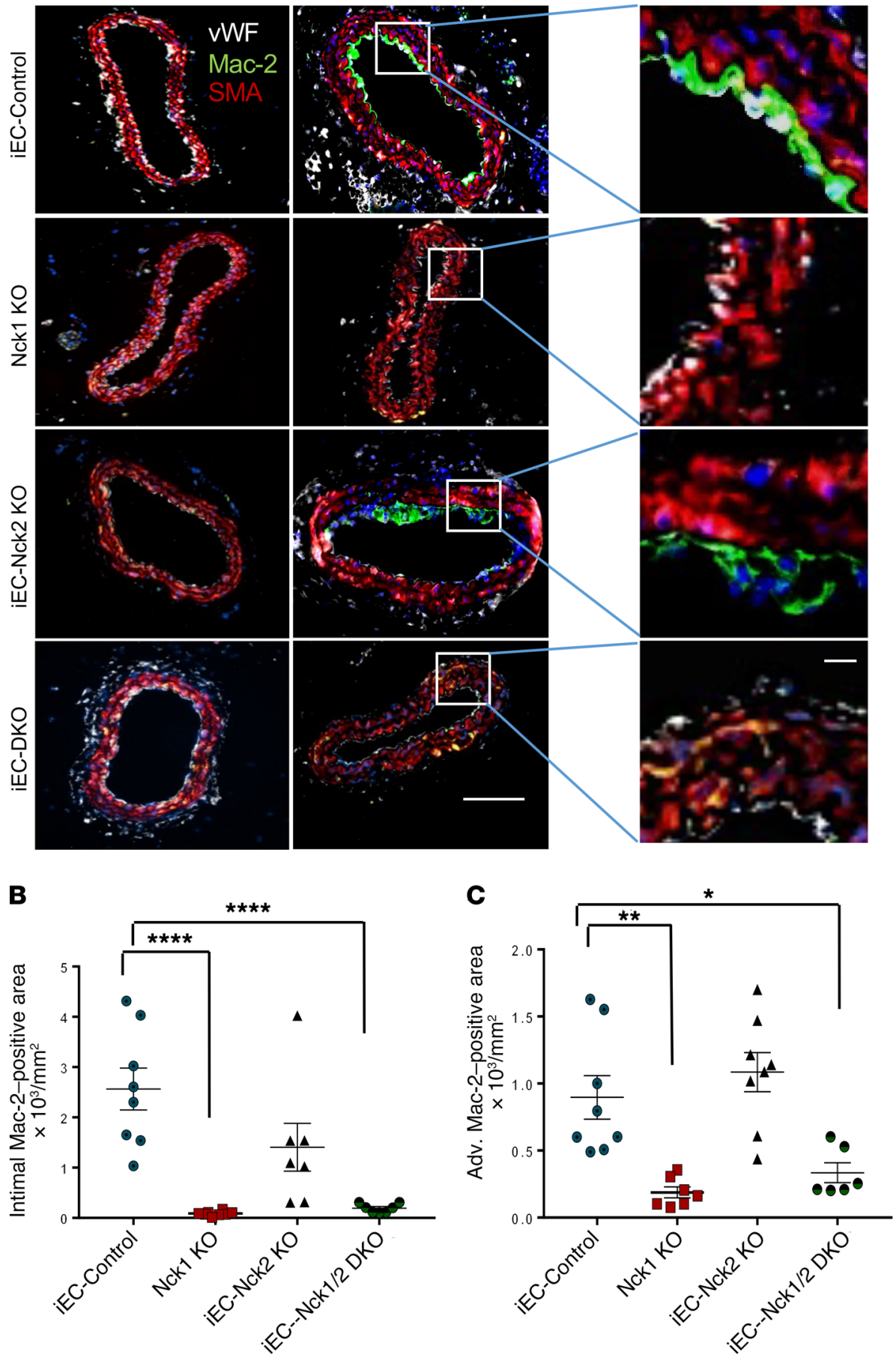

Figure 4. Nck1 deletion reduces macrophage infiltration in the partial carotid ligation model of disturbed flow. (A) Macrophages (Mac $-2^{+}$, green) in the ligated and the unligated carotid arteries among experimental groups. Smooth muscle cells ( $\alpha$-smooth muscle actin ${ }^{+}\left[\mathrm{SMA}^{+}\right]$, red), and endothelium (vWF', white). Scale bars: $200 \mu \mathrm{m}$ (left 2 columns) and $50 \mu \mathrm{m}$ (right column). (B and C) Graphical quantification of intimal and adventitial Mac-2-positive cells. Images analyzed using NIS Elements software, from $n$ $=7-10$ mice/group. Data are the mean \pm SEM. ${ }^{*} P<0.05$; ${ }^{* *} P<0.01$; ${ }^{* * *} P<0.0001$ by 1 -way ANOVA followed by Tukey's post hoc test.

staining was markedly enhanced in the ligated carotids, predominantly within the endothelium (Figure 8H). However, endothelial cell IRAK-1 phosphorylation was significantly abolished in Nck1-KO and Nck1/2-DKO mice (Figure 8, H and I). Consistent with these data, brachiocephalic atherosclerotic plaques showed enhanced phospho-IRAK-1 staining in the endothelium that was markedly reduced in Nck1-KO mice (Supplemental Figure 12), confirming the role of Nck1 in IRAK-1 activation at atherosclerosis-prone sites.

Nck1 and IRAK-1 contribute to early atherogenic endothelial activation. To determine if IRAK-1 contributes to disturbed flow-induced NF- $\mathrm{B}$ activation, IRAK-1 was depleted in HAECs using siRNA SMARTPool treatment (Figure 9A), and the cells were exposed to OSS for 18 hours. IRAK-1 knockdown significantly blunted NF- $\mathrm{KB}$ activation and proinflammatory gene expression in response to OSS (Figure 9, A and B). Finally, we assessed Nck1 and phospho-IRAK-1 staining in endothelial cells overlying early (stage 1) human atherosclerotic plaques. Both Nck1 and phospho-IRAK-1 showed enhanced staining in endothelial cells associated with early atherosclerotic plaques in humans (Figure 9C) but not

activation of IRAK-1 (Ser/Thr209 phosphorylation). Although we did not observe any significant changes in Nck1 and IRAK-1 total protein levels, cells exposed to atheroprotective laminar flow showed marked reduction in IRAK-1 phosphorylation, whereas those exposed to the atheroprone OSS showed marked induction of IRAK-1 phosphorylation (Figure 8, D and E), suggesting that OSS promotes IRAK-1 activation. To test whether IRAK-1 activation is Nck1 dependent (Figure 8F), Nck1-depleted HAECs were subjected to OSS, resulting in a marked reduction in IRAK-1 phosphorylation (Figure 8G). Following PCL in vivo, phospho-IRAK-1 healthy human coronary sections (Figure 9D).

Collectively, our data reveal a role for Nck1 in endothelial activation by atheroprone hemodynamics and demonstrate a link between Nck1 and IRAK-1 activation in mediating disturbed flowinduced endothelial activation.

\section{Discussion}

Atherogenic endothelial activation promotes vascular permeability and enhances adhesiveness for circulating leukocytes (29). In early atherosclerosis, atheroprone disturbed flow (low 
A

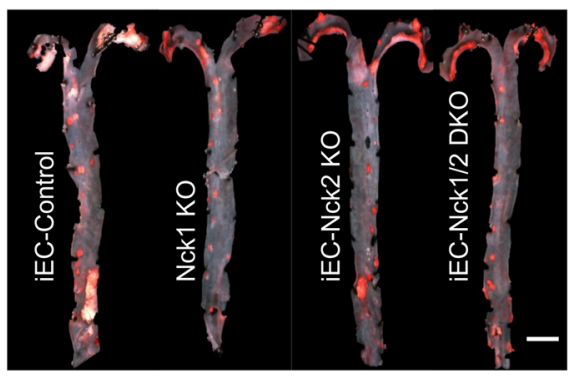

B
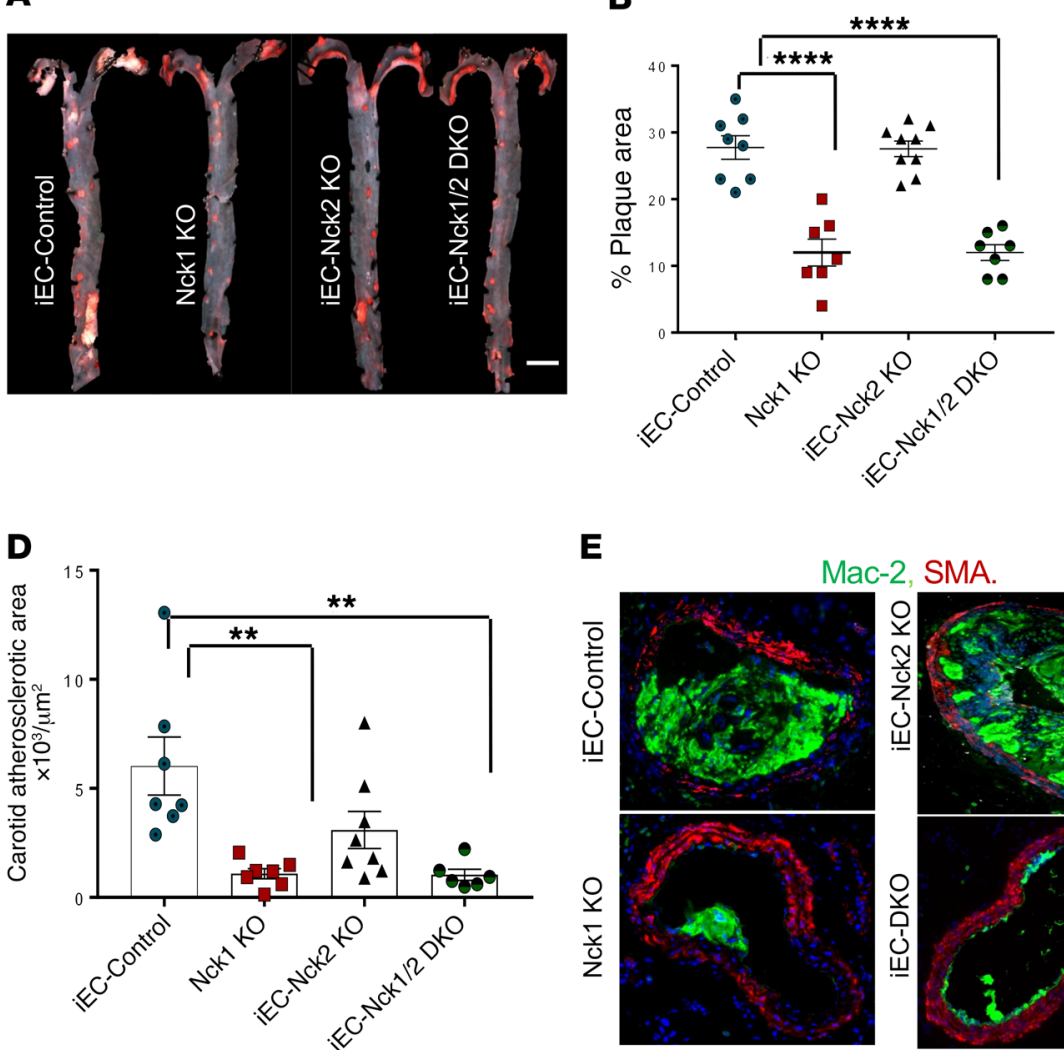

G
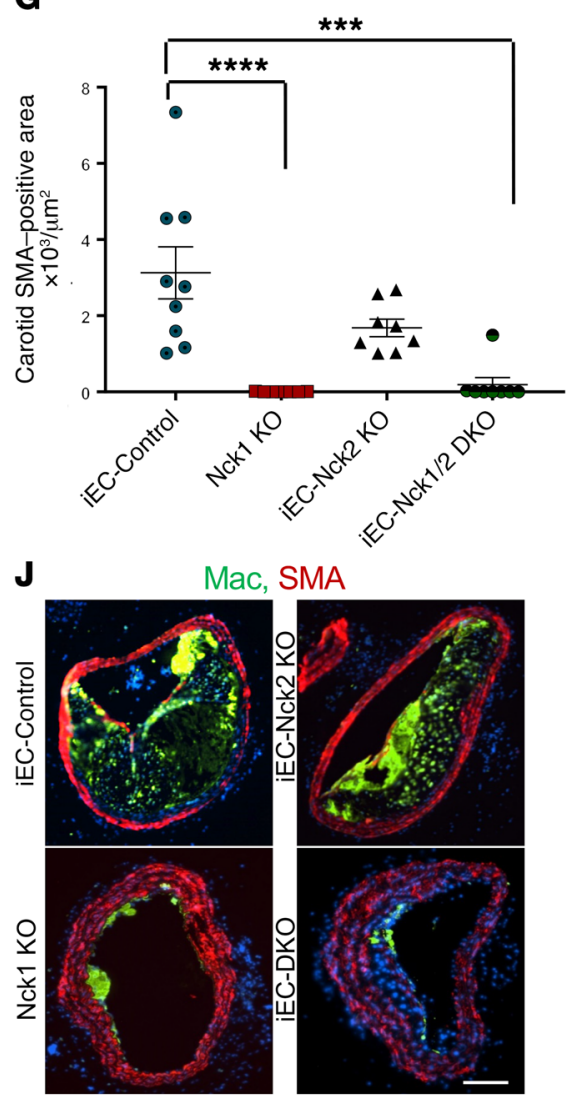

E

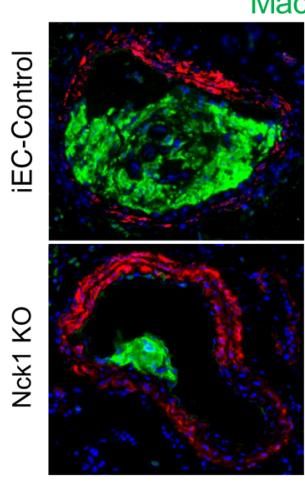

H

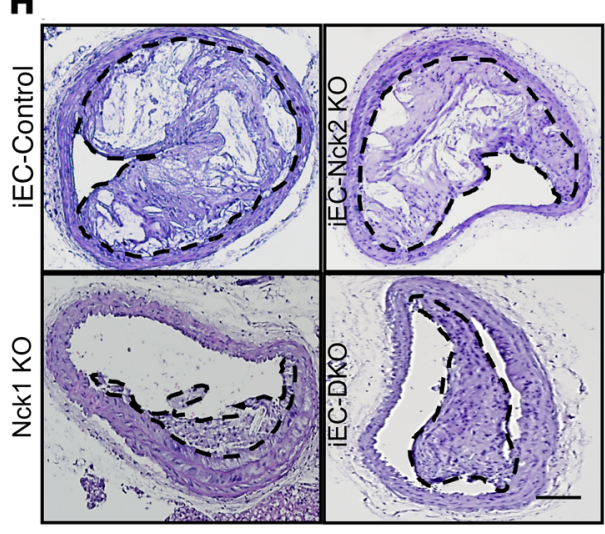

K

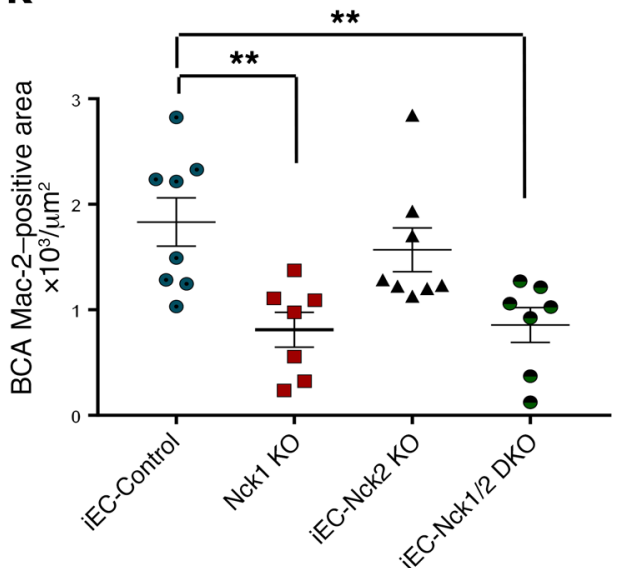

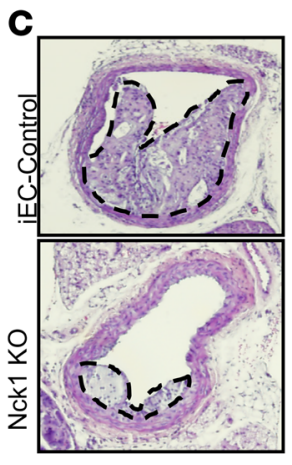

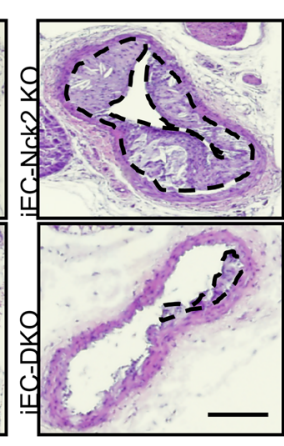

F
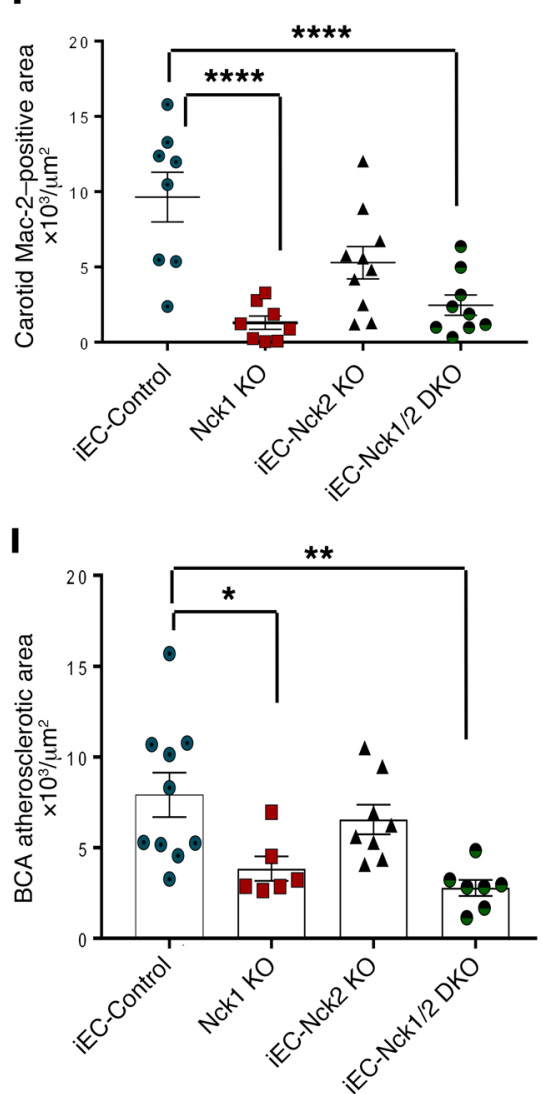

$\mathbf{L}$

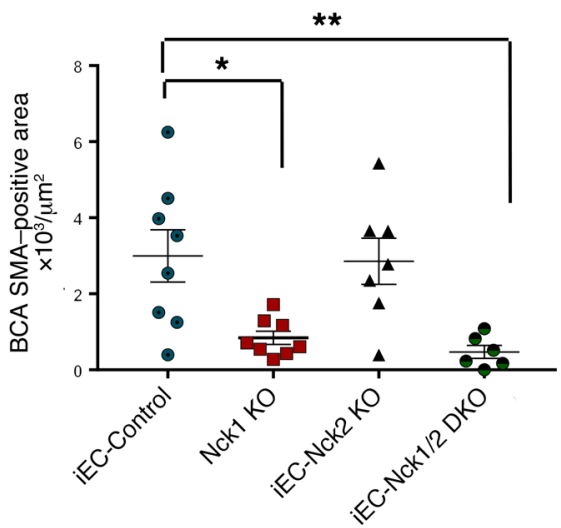


Figure 5. Global deletion of Nck1, but not Nck2, blunts high-fat dietinduced atherosclerosis. iEC-Control, Nck1-KO, iEC-Nck2-KO, and iECNck1/2-DKO mice were fed high-fat diet (HFD) for 12 weeks. (A) Representative en face morphometric images of total aortic lesion area and (B) calculated whole aortic atherosclerosis (percentage of the total surface area). Scale bar: $1 \mathrm{~mm}$. (C) Representative images of hematoxylin and eosin-stained (H\&E-stained) atherosclerotic carotid and (D) quantification of carotid atherosclerotic area among experimental groups. (E-C) Analysis of carotid plaque cellular content following staining for macrophages (Mac- $2^{+}$, green), and smooth muscle cells ( $\alpha$-smooth muscle actin ${ }^{+}\left[\mathrm{SMA}^{+}\right]$, red). (H) H\&E staining of brachiocephalic arteries (BCAs) after HFD feeding in iEC-Control, Nck1-KO, iEC-Nck2-KO, and iEC-DKO mice. (I) Quantification of atherosclerotic burden in BCAs among experimental groups. (J) Plaque composition assessed by staining for macrophages (Mac-2+) and smooth muscle cells $\left(\mathrm{SMA}^{+}\right)$in brachiocephalic lesions. (K) Quantification of Mac- $2^{+}$ and (L) SMA ${ }^{+}$areas in BCAs. Data are the mean \pm SEM, $n=6-10 /$ group. ${ }^{*} P$ $<0.05$; ${ }^{*} P<0.01$; ${ }^{* *} P<0.001$; ${ }^{* * *} P<0.0001$ by 1-way ANOVA followed by Tukey's post hoc test. Scale bars: $100 \mu \mathrm{m}$ (C, E, H, and J).

magnitude blood flow with complex features including turbulence, oscillations, separation, and reattachment) promotes endothelial activation (2). Elucidating the pathogenic mechanism of disturbed flow-induced endothelial activation in early lesion formation may lead to interventions that delay or even prevent lesion progression and complications. In this study, we demonstrate what we believe is a novel, isoform-specific role for Nck1 in atherogenic endothelial activation in vitro and in vivo. We show that Nck1 is a critical signaling mediator of disturbed flow-induced NF- $\mathrm{KB}$ activation and proinflammatory gene expression, and we demonstrate important roles for the Nck1 SH2 domain and first SH3 domain in mediating this effect. We provide the first validation to our knowledge that IRAK-1 serves as a Nck1-specific binding protein, show a vital role for Nck1 in flow-induced IRAK-1 activation, and demonstrate that IRAK-1 is required for chronic NF- $\mathrm{KB}$ activation by disturbed flow. Although highly similar to Nck1, endothelial Nck2 expression is dispensable for flow-induced endothelial activation. In vivo, only vascular Nck1 deletion reduces endothelial proinflammatory gene expression, monocyte recruitment, and early plaque formation in both the PCL model of disturbed flow and in diet-induced spontaneous atherosclerosis, and both Nck1 and phospho-IRAK-1 show enhanced staining in early human atherosclerotic plaques. Taken together, these data clearly identify an isoform-specific role for Nck1 in mediating endothelial activation under atheroprone hemodynamics.

The Nck adaptor proteins have been extensively studied in diverse signaling events, most often affecting pathways leading to cellular morphogenesis (10). Previous studies have identified at least 60 Nck1- and Nck2-associated proteins, mostly involved in cytoskeletal organization $(23,33)$. In models of cell migration and tissue remodeling, Nck1 and Nck2 appear to be largely redundant. Deletion of both Nck1 and Nck2 during development hinders vasculogenesis, whereas individual deletion of either Nck1 or Nck2 did not (22). In the ischemic retinopathy model, deletion of both Nck1 and Nck2 were required to reduce angiogenesis associated with diminished endothelial cell and pericyte migration $(12,34)$. However, Guan et al. (35) demonstrated that Nck1 and Nck2 can act independently in dermal fibroblasts' migration and $\mathrm{T}$ cell activation (14). We previously demonstrated that depletion of both Nck1 and Nck2 by siRNA blunts oxidative stress-induced NF- $\kappa B$ activation in models of ischemia/reperfusion injury (15), but isoform-specific roles were not addressed. Our current data provide the first direct evidence to our knowledge that Nck1 meditates inflammation due to atheroprone flow and demonstrate an unexpected isoform-selective role in mediating disturbed flowinduced NF- $\mathrm{KB}$ activation and proinflammatory gene expression within the endothelium.

Composed exclusively of $\mathrm{SH} 2 / \mathrm{SH} 3$ domains, Nck proteins have no enzymatic activity but regulate multiple signal transduction pathways by controlling protein localization and signaling complex formation $(10,23)$. Although the modular structure of Nck1/2 allows for numerous individual and probably simultaneous protein-protein interactions (11), our data specifically identify a role for the Nck1 SH2 domain and a redundant role for the first Nck1/2 SH3 domain as the critical sites mediating endothelial activation by atheroprone flow. Despite the fact that the consensus SH2 binding sequences for Nck1 and Nck2 are highly similar (36), the Nck1 and Nck2 SH2 domains are able to bind distinct phosphotyrosine sequences on growth factor receptors (36) and at sites of cell adhesion (36). Consistent with this selectivity, phosphorylated Nephrin selectively recruits Nck1 and not Nck2 through Nck1's SH2 domain in vivo (31). To our knowledge the binding and signaling properties of individual Nck1 SH3 domains have yet to be systematically explored. The critical SH3.1 domain in Nck1 binds an atypical PXXDY motif that undergoes negative regulation by tyrosine phosphorylation, providing a potential negative feedback response that would be expected to limit atherogenic endothelial activation. However, future studies will be required to identify the Nck1 SH3.1 binding partners important for endothelial activation by atheroprone flow.

To explore the noncompensating role of the Nck1 SH2 and SH3.1 domains in disturbed flow-induced endothelial activation, we sought to identify potential binding partners that could mediate endothelial activation by disturbed flow. A previous report using the BioID proximity biotinylation system to identify Nck1 and Nck2 binding proteins found IRAK-1 as a specific interacting partner for Nck1 (25). Although this interaction was proposed to be $\mathrm{SH} 2$ dependent using computer modeling software, the specific domain mediating this interaction was not determined. Our coimmunoprecipitation studies confirm the direct interactions between IRAK-1 and Nck1, but not Nck2, and demonstrate enhanced interactions following exposure to disturbed flow.

IRAK-1 was first identified through its association with IL-1 receptors (IL-1Rs) at the plasma membrane (32) and is a critical mediator of IL-1-induced NF-кB activation (37). Ubiquitously expressed, IRAK-1 has been extensively studied in immune cells (38) and is activated in response to Toll-like receptor (TLR) and IL-1R signaling. Interestingly, IRAK-1 activation is critically dependent on T209 phosphorylation (39), and T209 was highly phosphorylated in HAECs exposed to OSS. Activation of TLRs and IL-1Rs induces binding of MyD88, a signaling scaffold that recruits IRAK-1 to promote its maximum activation and couple it to downstream signaling partners (37). Although Nck1 and MyD88 have disparate domain structures, Nck1 may similarly mediate IRAK-1 recruitment to activated cell surface receptors 

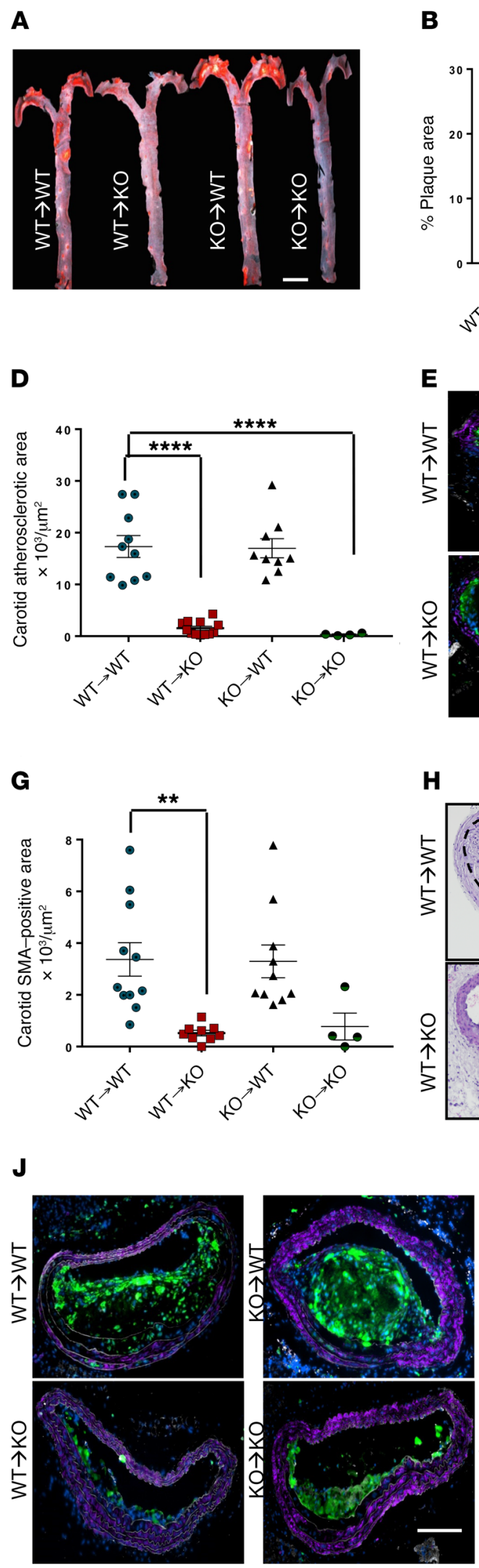

SMA, Mac2
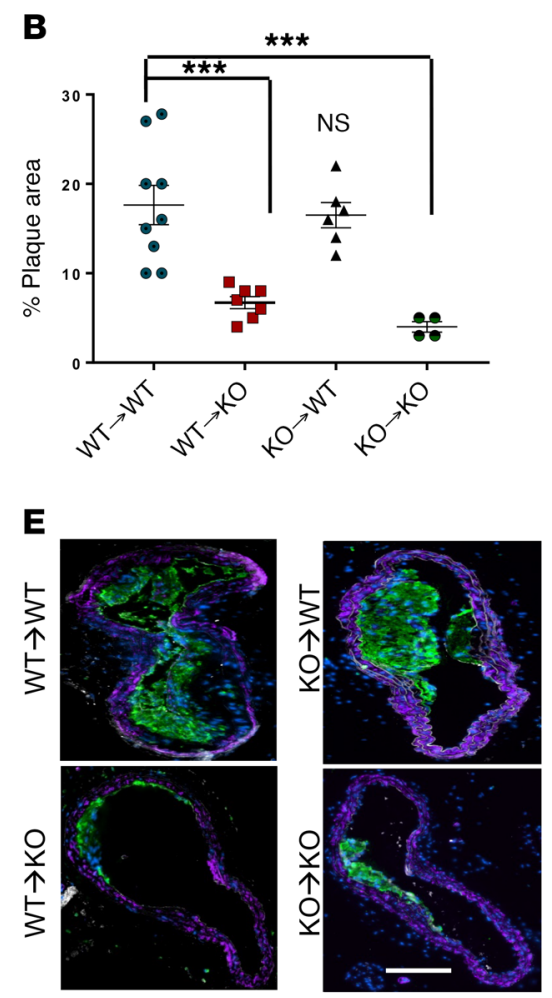

SMA, Mac2
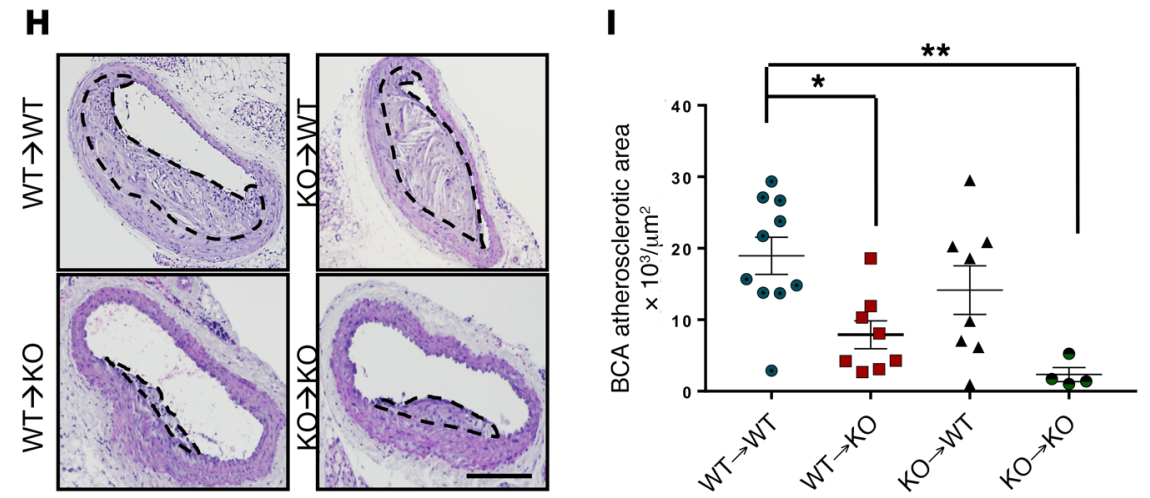

$$
\mathbf{L}
$$
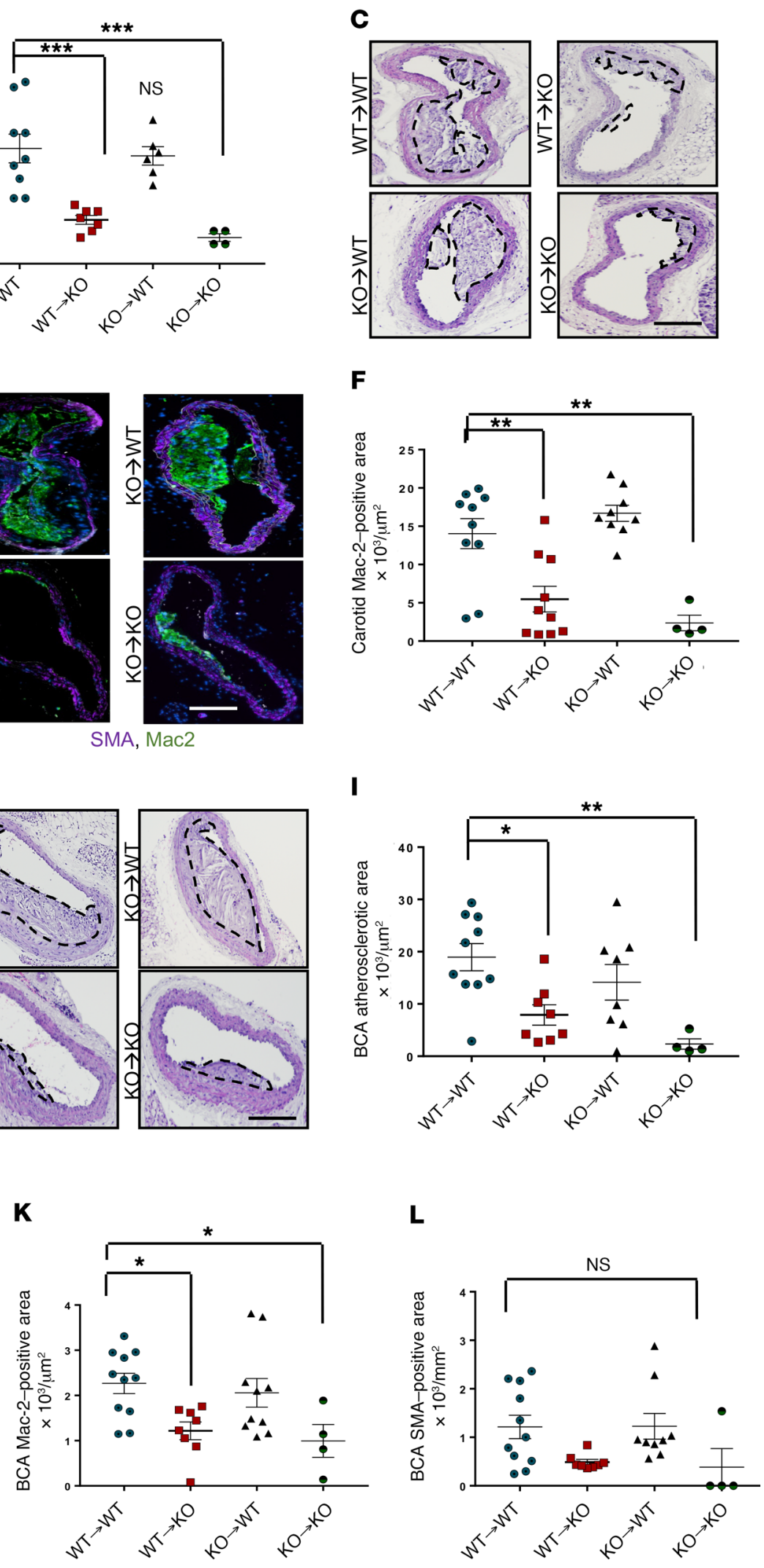
Figure 6. Nck1 deletion from vascular wall, but not hematopoietic cells, has atheroprotective effect. Nck1-WT (WT) and Nck1-KO mice were irradiated and received bone marrows from either Nck1-WT or Nck1-KO mice. Two weeks after irradiation, the 4 groups of mice were fed HFD for 12 weeks. (A) Representative en face morphometric images of total aortic lesion area and (B) calculated whole aortic atherosclerosis (percentage of the total surface area). Scale bar: $1 \mathrm{~mm}$. (C) Representative images of H\&E-stained atherosclerotic carotid artery and (D) quantification of carotid atherosclerotic area among experimental groups. (E-G) Analysis of plaque cellular content following staining for macrophages (Mac- $2^{+}$, green), and smooth muscle cells ( $\alpha$-smooth muscle actin ${ }^{+}\left[\mathrm{SMA}^{+}\right]$, purple). (H) H\&E staining of brachiocephalic arteries (BCAs) after HFD feeding in WT $\rightarrow \mathrm{WT}, \mathrm{WT} \rightarrow \mathrm{KO}, \mathrm{KO} \rightarrow \mathrm{WT}$, and Nck1-KO $\rightarrow$ Nck1-KO mice. (I) Quantification of atherosclerotic burden in BCAs among experimental groups. (J) Plaque composition assessed by staining for macrophages (Mac- $2^{+}$) and smooth muscle cells $\left(\mathrm{SMA}^{+}\right)$in brachiocephalic lesions. (K) Quantification of Mac- $2^{+}$and (L) SMA ${ }^{+}$areas in BCAs. Data are the mean \pm SEM, $n=4-11 /$ group. ${ }^{*} P<0.05 ;{ }^{* *} P<0.01$; ${ }^{* * *} P$ $<0.001 ;{ }^{* * *} P<0.0001$ by 1 -way ANOVA and Tukey's post hoc test. NS, not significant. Scale bars: $100 \mu \mathrm{m}$ (C, E, H, and J).

and facilitate its coupling to downstream signaling components to activate NF- $\mathrm{kB}$ by disturbed flow. Indeed, phospho-IRAK-1 was reduced when Nck1 was deleted in our in vitro and in vivo systems, suggesting that in endothelial cells Nck1 is an upstream mediator of IRAK- 1 activation.

Significantly, phospho-IRAK-1 and Nck1 show enhanced staining within the endothelium in early human atherosclerosis. In line with this finding, a genome-wide association study in 34,541 coronary artery disease patients of European biobanks identified Nck1 as a novel coronary artery disease susceptibility locus (18). To our knowledge, these data provide the first description of Nck1's potential role in regulating this enhanced susceptibility. However, the pathways upstream of disturbed flow-induced Nck1/IRAK-1 signaling remain unknown. Potential activators of Nck1/IRAK-1 signaling include direct recruitment and activation by mechanosensitive signaling pathways or indirect activation by secretion of IL-1R/TLR ligands. Other groups have shown inflammasome activation and IL-1 $\beta$ processing in response to disturbed flow (40), and disturbed flow-induced TLR4 signaling has been shown to correlate with enhanced deposition of fibronectin containing the EIIIA domain predicted to bind TLR4 (41). Interestingly, both disturbed flowinduced inflammasome activation and induction of fibronectin EIIIA deposition require the fibronectin-binding integrin $5 \beta 1$ $(40,42)$. However, additional research will be needed to identify the upstream pathways mediating Nck1/IRAK-1 activation in response to disturbed flow.

Owing to its known role in vasculogenesis and angiogenesis, Nck1/2 signaling was previously viewed as a poor target to treat atherosclerotic cardiovascular disease. A blocking peptide mimicking the Nck1/2 SH3.2 (second SH3) domain reduces angiogenesis in vivo (43), blunts vascular permeability in models of ischemia/reperfusion injury (15) and atherosclerosis (44), and limits inflammation in atherosclerosis and LPS-induced lung injury $(39,40)$. However, targeting both Nck1 and Nck2 with this inhibitor is unlikely to be beneficial in treating atherosclerotic disease, as the inhibitor would limit angiogenesis in ischemic regions affected by the plaque. Our findings suggest the possibility of selectively targeting Nck1 to limit plaque-associated inflam- mation without hindering angiogenesis in ischemic tissue, as Nck2 can compensate for Nck1 in this regard. Because inhibition of either isoform alone is not sufficient to reduce angiogenesis $(12,34)$, targeting Nck1 may represent a realistic therapeutic strategy to limit endothelial activation without affecting angiogenic tissue remodeling.

In conclusion, our results provide the first data to our knowledge linking endothelial Nck1 signaling to atherogenic endothelial activation and atherosclerotic plaque development. Furthermore, we identified the Nck1 SH2 domain and IRAK-1 as critical mediators of atheroprone flow-induced NF-kB activation and proinflammatory gene expression. Taken together, our findings extend our current understanding of endothelial cell activation in response to atheroprone hemodynamics and identify inhibition of Nck1 as a potential future therapeutic approach to treating atherosclerotic cardiovascular disease.

\section{Methods}

Cell culture, plasmids, and RNA interference. HAECs (CELL Applications) were maintained in MCDB131 containing 10\% (v/v) fetal bovine serum and supplemented with bovine brain extract $(24 \mu \mathrm{g} /$ $\mathrm{mL}$ ), $2 \mathrm{mmol} / \mathrm{L}$-glutamine, $10 \mathrm{U} / \mathrm{mL}$ penicillin, and $100 \mu \mathrm{g} / \mathrm{mL}$ streptomycin. Cells were used between passages 6 and 10. HAEC transformation was induced by transduction of HAECs with a lentiviral vector expressing SV-40 T-antigen, as previously reported

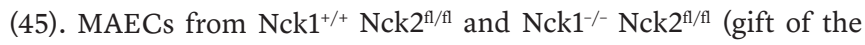
late Tony Pawson, Samuel Lunefeld Research Institute, Toronto, Canada) were isolated from aortic rings as previously described (46) and the cells were kept in MCDB131 containing 10\% (v/v) media. To induce Nck2 KO or DKOs, the cells were infected with adenovirus expressing either GFP $\left(1 \times 10^{8} \mathrm{PFU} / \mathrm{mL}\right)$ or GFP-2A-iCre (improved mammalian expression, conjugated to GFP, $2 \times 10^{8} \mathrm{PFU} / \mathrm{mL}$; Vector Biolabs) and sorted for GFP positivity. For shRNA delivery, the lentiviral vector used was $\mathrm{pLV}$-(shRNA)-mCherry:T2A:Puro-U6 (Nck1 target sequence: GGGTTCTCTGTCAGAGAAA; Nck2 target sequence: CTTAAAGCGTCAGGGAAGA; VectorBuilder) with third-generation lentiviral components provided from Addgene; pMD2.G (plasmid 12259), pRSV-Rev (plasmid 112253), pMDLg/ pRRE (plasmid 112251); all were deposited by Didier Trono (47). The resulting plasmids were used to package lentiviruses and infect target cells. Transfection with SMARTpool siRNAs targeting Nck1 (L-006354, IDT) and Nck2 (L-019547, IDT) was performed using Lipofectamine 3000 (Invitrogen) according to the manufacturer's recommendations. CRISPR/Cas9 deletion of Nck1 and Nck2 used double-guide RNA (dgRNA) sequences targeting Nck1 (GTCGTCAATAACCTAAATAC), Nck2 (TGACGCGCGACCCCTTCACC), or scrambled sgRNA (GCACTACCAGAGCTAACTCA). The lentiviral vectors encoding the full-length Nck1 and Nck2 were generated in the pLV-EXP-mCherry:T2A:Puro-CMV>Nck vector. The domainswap experiments used pLV-EXP-mCherry:T2A:Puro-CMV>Nck constructs containing Nck1 SH2 and Nck2 SH3 domains or Nck2 SH2 and Nck1 SH3 domains. Point mutations in Nck1 SH2 and SH3 domains were generated by the Redox Molecular Signaling Core (COBRE Center for Cardiovascular Diseases and Sciences, LSU Health Sciences Center, Shreveport, Louisiana, USA). Nck1 domain point mutations were verified by cloning PCR and sequencing (Eurofins Genomics) before being used for experiments. 
A

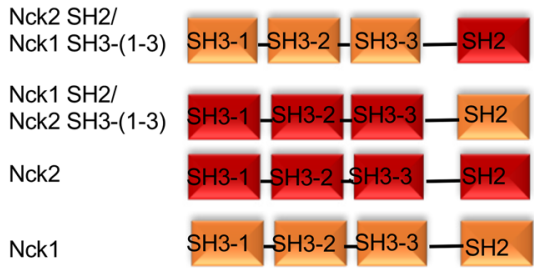

D

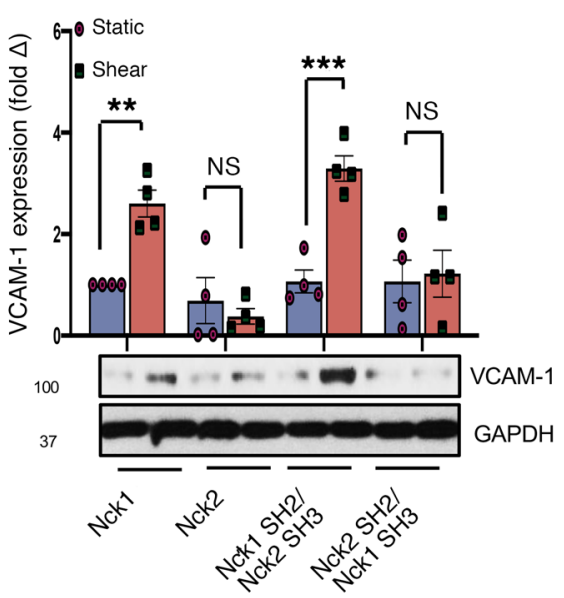

$\mathbf{G}$

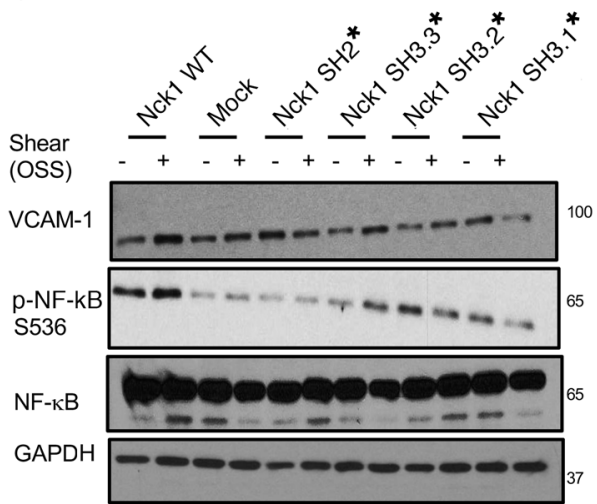

B

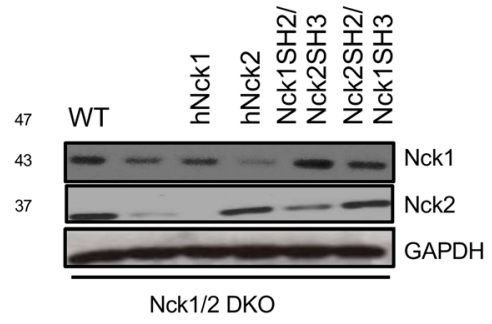

E

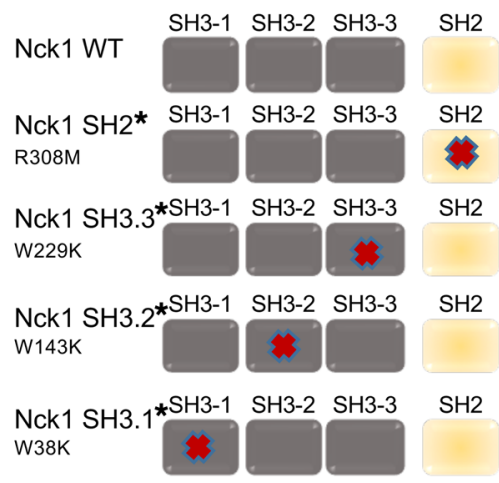

C

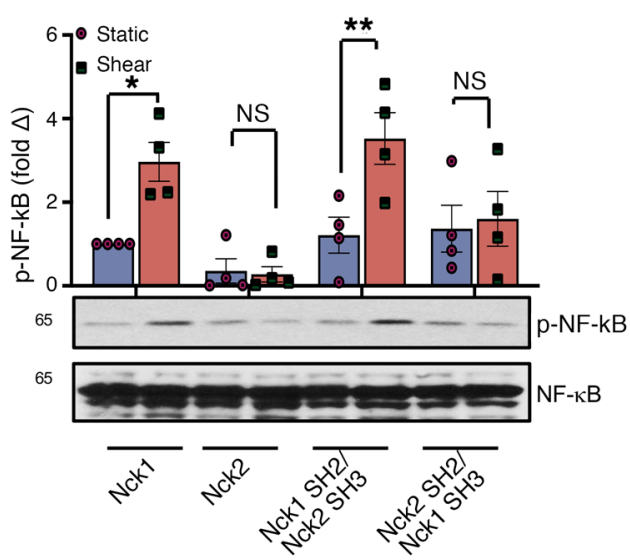

$\mathbf{F}$

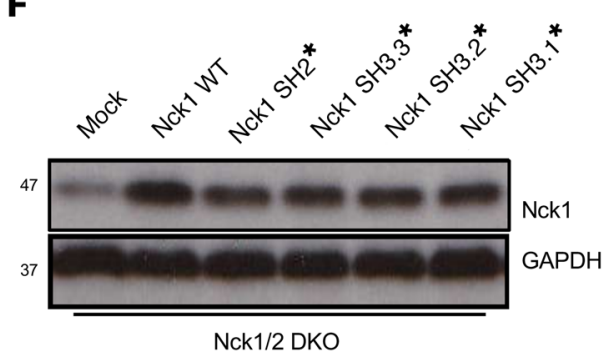

H

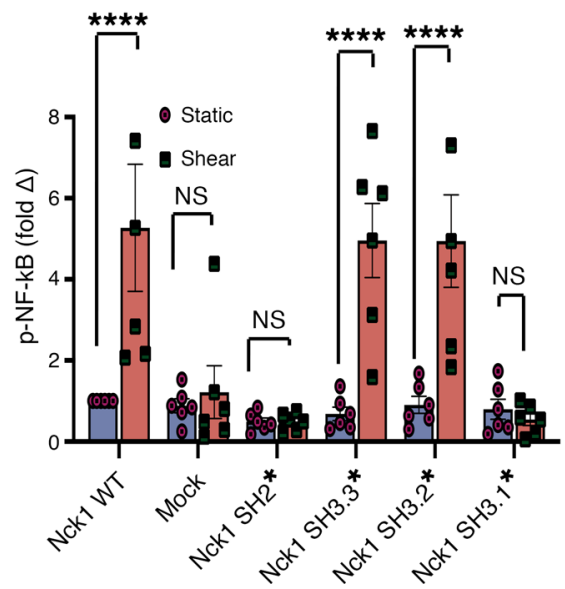

I

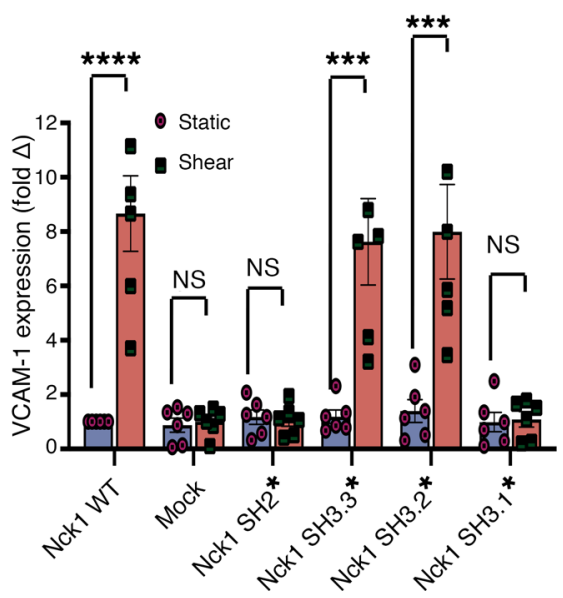

Figure 7. Nck1 regulates shear stress-induced inflammation via its SH2 domain and the first of its $\mathbf{3}$ SH3 domains. (A) Schematic showing the domain structure of Nck1 and Nck2 and the 2 chimeras consisting of Nck1 SH2/Nck2 SH3.1-SH3.3 and Nck2 SH2/Nck1 SH3.1-SH3.3. (B) Western blot analysis showing comparable transduction efficiency of the Nck1/2 chimeras after transducing the constructs into Nck1/2-DKO cells. $n=3$. (C and D) Nck1/2-DKO HAECs were transduced with the constructs in $\mathbf{A}$, and OSS-induced proinflammatory signaling (p-NF-KB Ser536) and gene expression (VCAM-1) were assessed. (E) Schematic of Nck1 SH3 domain point mutations, and (F) Western blot analysis showing the efficiency of reexpression of different Nck1 mutants in Nck1/2DKO HAECs. (G-I) Nck1/2-DKO HAECs were transiently transfected with Nck1 or Nck1 variants described in E, and 0SS-induced proinflammatory signaling and gene expression assessed as indicated above. Data are from $n=4-6$ and presented as the mean $\pm \mathrm{SEM}$. ${ }^{*} P<0.05 ;{ }^{* *} P<0.01$; ${ }^{* * *} P<0.001 ;{ }^{* * *} P<$ 0.0001 by 2-way ANOVA followed by Bonferroni's post hoc test. NS, not significant.

Induction of acute-onset and chronic shear stress in vitro. Shear stress experiments were performed using parallel-plate flow chambers, as we previously published $(48,49)$. To test the early onset of the proinflammatory endothelial activation, cells were subjected to acute shear stress $\left(12\right.$ dynes $\left./ \mathrm{cm}^{2}\right)$ for up to 45 minutes. Static cell culture was used as a negative control. OSS was induced using a syringe pump ( \pm 5 dynes $/ \mathrm{cm}^{2}$ with a superimposed $1 \mathrm{dyne} / \mathrm{cm}^{2}$ for waste exchange) for 18 hours. Chronic laminar shear stress was induced by subjecting the cells to 12 dynes $/ \mathrm{cm}^{2}$ for 18 hours. After cessation of flow, the cells were rapidly lysed using $2 \times$ Laemmli buffer for Western blot, TRIzol (Thermo Fisher Scientific) for qRT-PCR, or fixed in $4 \%(\mathrm{v} / \mathrm{v})$ formaldehyde for immunostaining.

Western blot. Proteins in the cell lysates were separated using SDS-PAGE gels and transferred to PVDF membranes (Bio-Rad). 
A

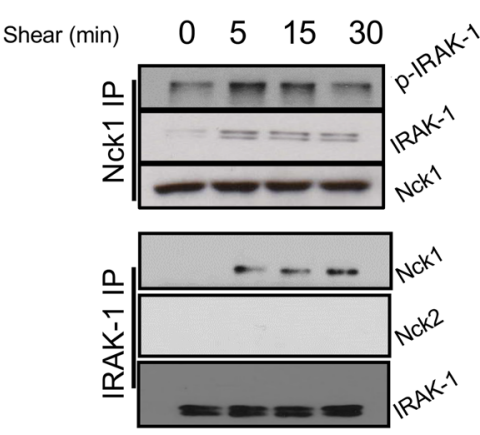

D

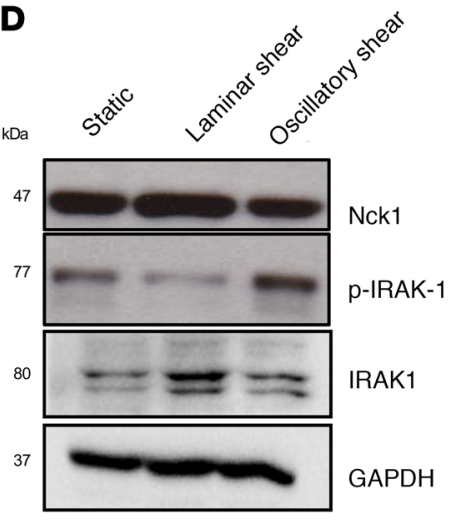

G
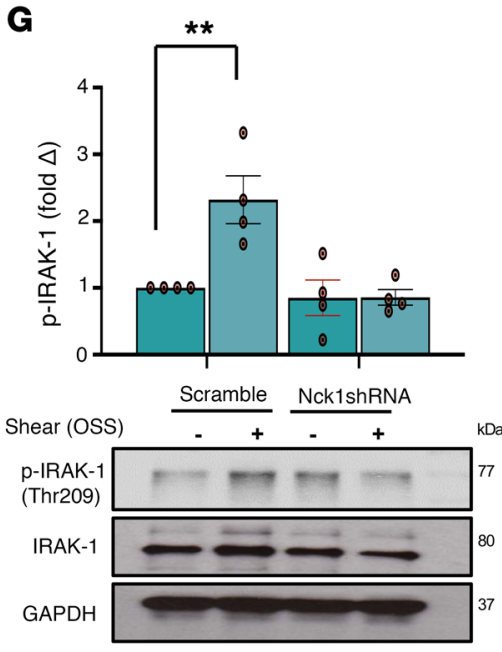

B

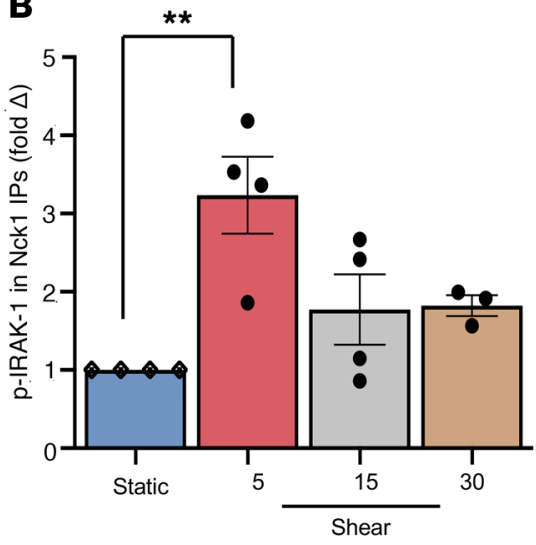

E

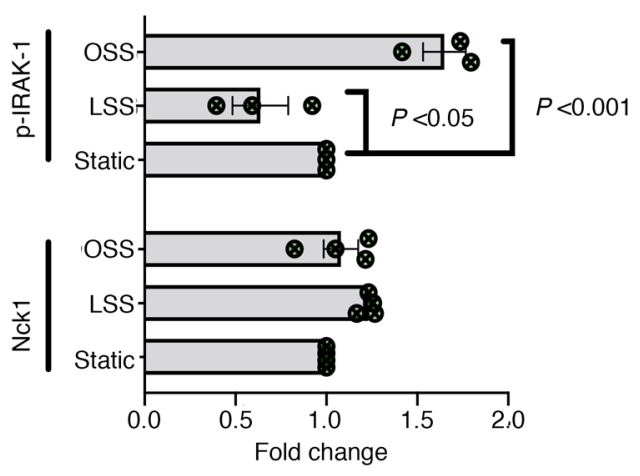

H

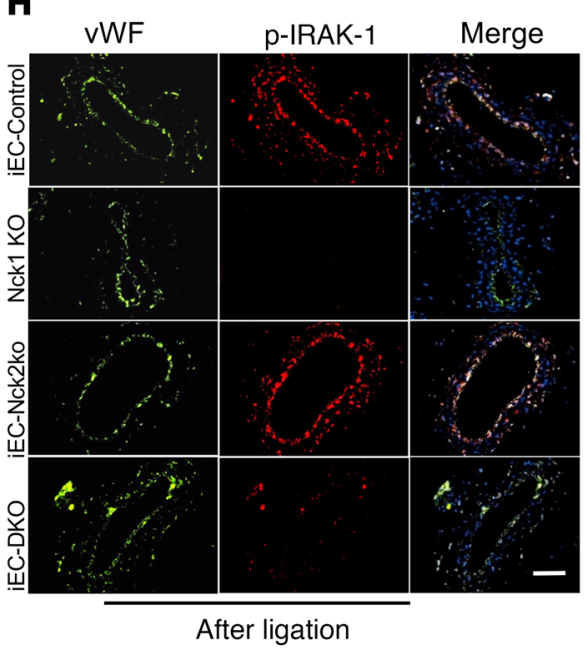

C

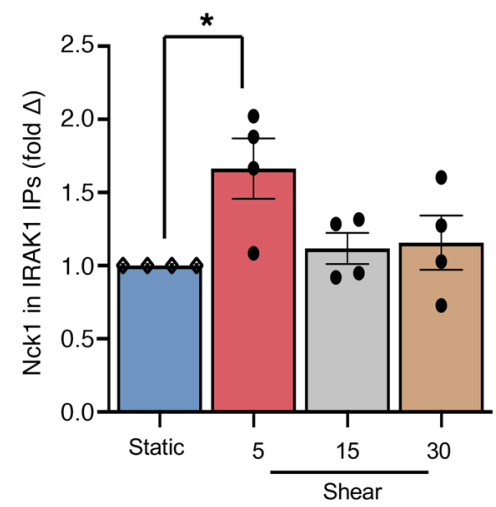

$\mathbf{F}$

Nck1
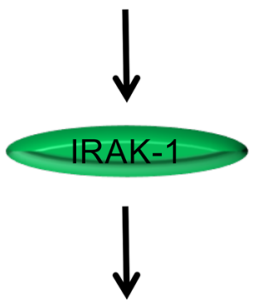

$\mathrm{NF}-\mathrm{KB}$

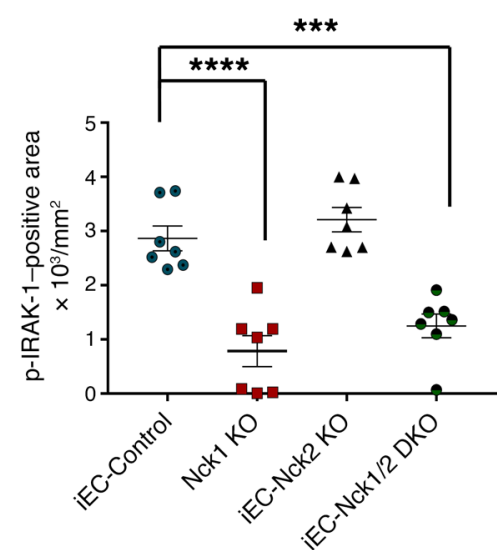

Figure 8. Nck1 interacts with IRAK1, and IRAK1 activation requires Nck1 in response to shear stress. (A) Immunoblotting showing Nck1 and IRAK-1 coimmunoprecipitation (Co-IP), confirming Nck1 and IRAK-1 interaction and that it is shear stress dependent. (B and C) Graphical representation of the levels of p-IRAK-1 in Nck-1 Co-IP and Nck1 levels in IRAK-1 Co-IP showing statistical significance after shear stress. Data are from $n=4$ and are presented as the mean \pm SEM. ${ }^{*} P<0.05,{ }^{* *} P<0.01$ by 1-way ANOVA and Tukey's post hoc test. ( $\mathbf{D}$ and $\mathbf{E}$ ) Human aortic endothelial cells (HAECs) were subjected to laminar shear stress (LSS; 10 dynes $/ \mathrm{cm}^{2}, 18$ hours) or oscillatory shear stress (OSS; \pm 5 dynes $/ \mathrm{cm}^{2}$ with $1 \mathrm{dyne} / \mathrm{cm}^{2}$ forward flow, 18 hours). Cell lysates were assessed using Western blotting for Nck1, p-IRAK-1, and IRAK-1 levels. GAPDH was used a loading control. Representative blots from $n=4$. Data were analyzed by 2-way ANOVA followed by Bonferroni's post hoc test. (F) Schematic representation of the pathway activation. (C) Lentiviral shRNA-transduced HAECs were subjected to shear stress ( \pm 5 dynes $/ \mathrm{cm}^{2}$ with 1 dyne $/ \mathrm{cm}^{2}$ forward flow, 18 hours). Cell lysates were assessed using Western blotting for $p-I R A K-1$ and IRAK-1 levels. GAPDH was used a loading control. Representative blots from $n=4$; data presented as the mean \pm SEM. ${ }^{* *} P<0.01$ by 2 -way ANOVA followed by Bonferroni's post hoc test. (H) Immunostained ligated left carotid arteries from iEC-Control, Nck1-KO, iEC-Nck2-KO, and iEC-Nck1/2-DKO mice. p-IRAK-1 (red), predominately within the endothelium ( $\mathrm{VWF}^{+}$, green), is reduced in Nck1-KO mice. Scale bar: $200 \mu \mathrm{m}$. (I) Graphical quantification showing the significance of the data from $\mathbf{H}$. Data presented as the mean \pm SEM. ${ }^{* * *} P<0.001 ;{ }^{* * *} P<0.0001$ by 1-way ANOVA and Tukey's post hoc test. 
A

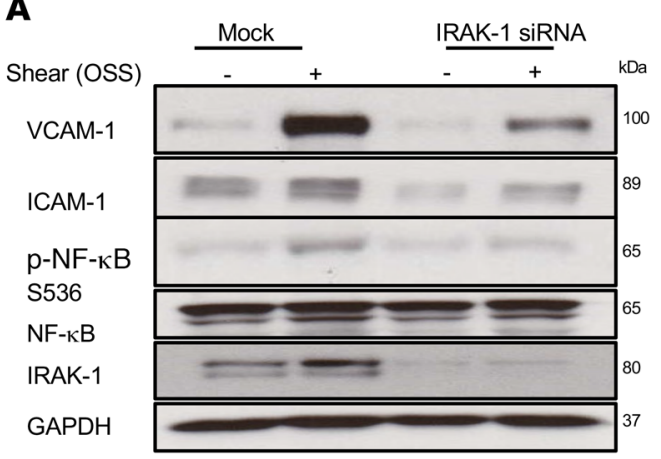

B

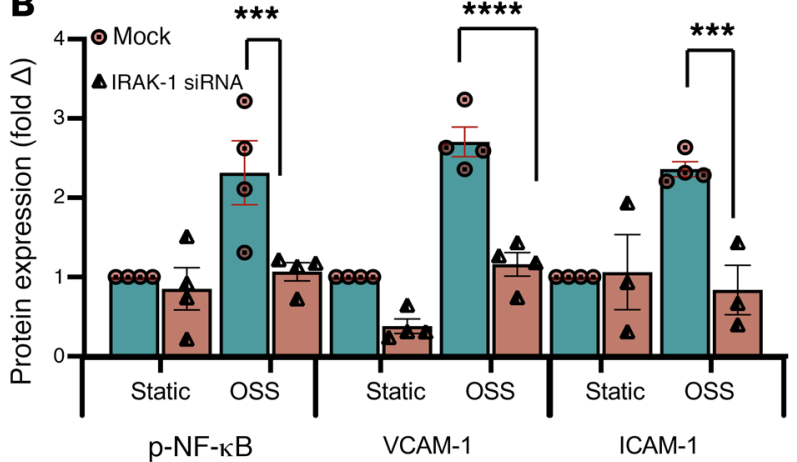

C

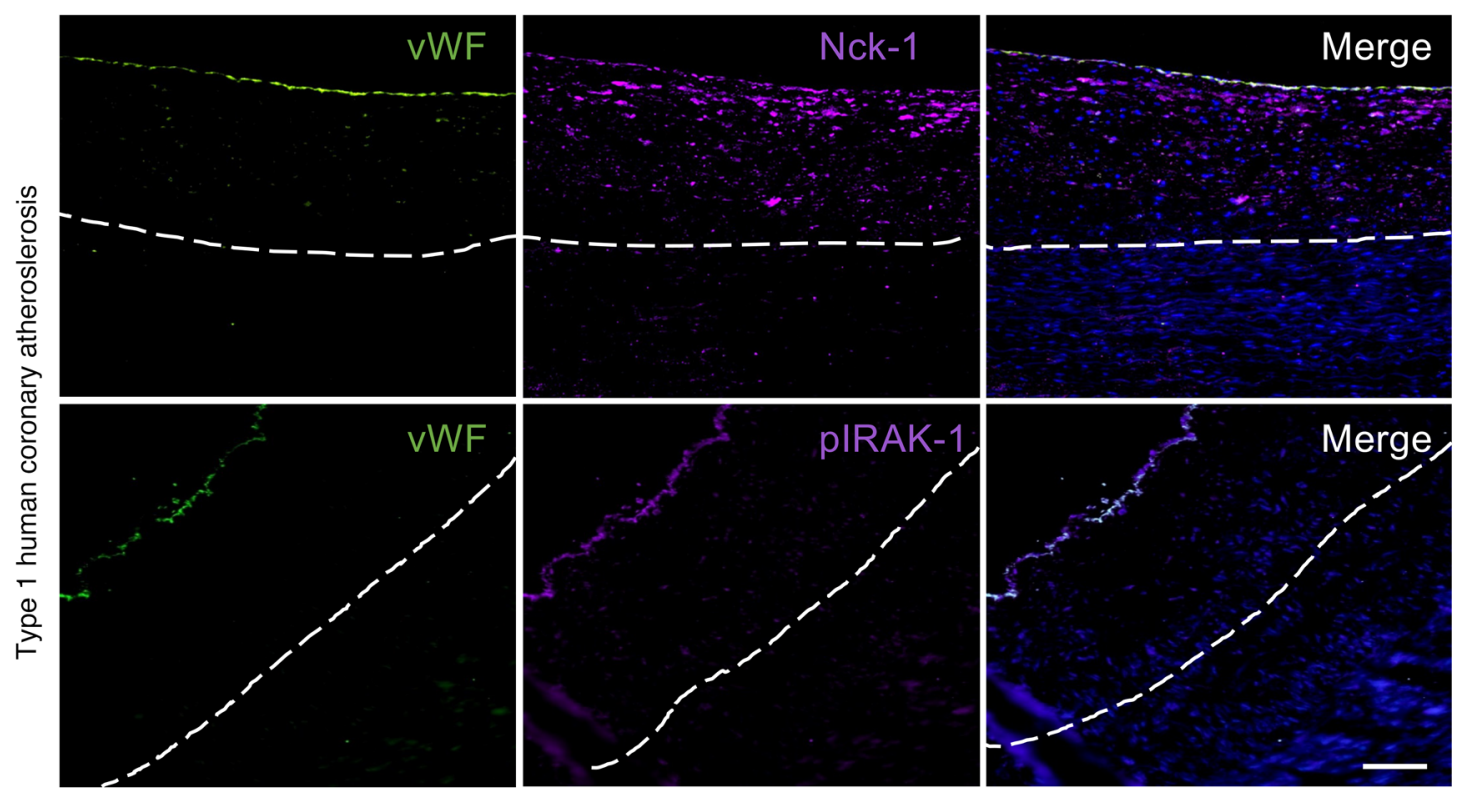

D
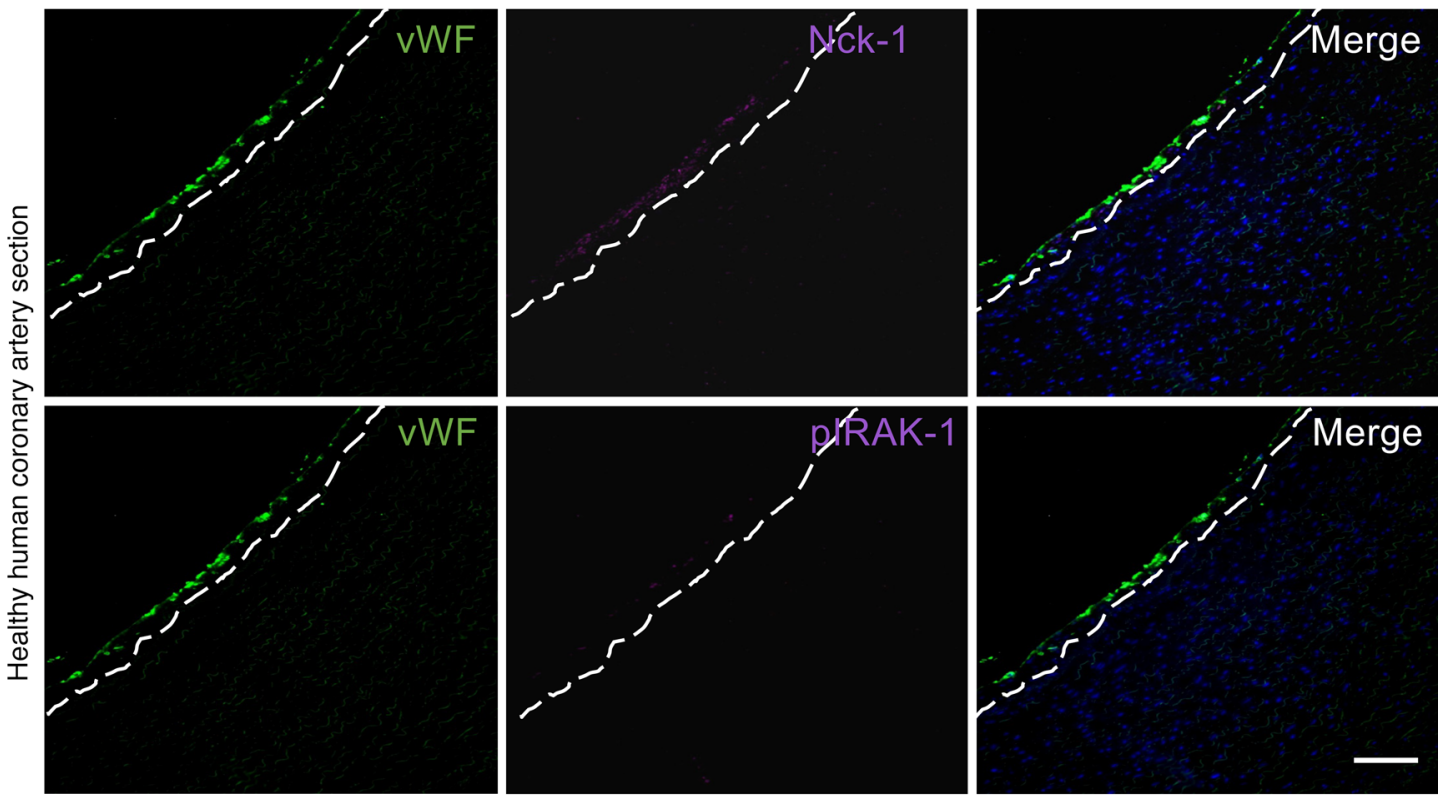

Figure 9. IRAK1 deletion ameliorates shear stress-induced activation. (A) HAECs were treated with or without IRAK-1 siRNA and subjected to oscillatory shear stress (OSS, 18 hours). Cell lysates were analyzed for VCAM-1, ICAM-1, and p-NF-אB using Western blotting. (B) Quantification of ICAM-1, VCAM-1, and $\mathrm{p}-\mathrm{NF}-\kappa \mathrm{B}$ showing significant reduction in IRAK-1-depleted cells. Densitometric analysis was performed using Image). Data are presented as the mean \pm SEM. ${ }^{* *} P<0.001,{ }^{* * *} P<0.0001$ by 2 -way ANOVA followed by Bonferroni's post hoc test. (C) Type I human atherosclerotic lesions were stained for p-IRAK-1 and Nck1, and vWF was used as an endothelial marker. (D) Healthy coronary sections were stained for p-IRAK-1, Nck1, and vWF. Dashed marks indicate internal elastic lamina. Scale bars: $100 \mu \mathrm{m}$. Images analyzed using NIS Elements software, from $n=5$ postmortem biopsy samples. 
After blocking nonspecific binding with $5 \%(\mathrm{w} / \mathrm{v})$ nonfat dry milk in TBS/0.1\% (v/v) Tween 20, the membranes were incubated with primary antibodies (see Supplemental Table 1 for details) at $4^{\circ} \mathrm{C}$ overnight. After secondary antibody incubation and chemiluminescence, densitometry was analyzed using NIH ImageJ software. See complete unedited blots in the supplemental material.

Quantitative real-time PCR. Total RNA was extracted from mouse carotid arteries using TRIzol as described previously (26), and HAECs of Nck1- and Nck2-knockdown and -KO cells. Reverse transcription was performed with an iScript kit (Bio-Rad, 1708890). qRT-PCR was performed using SYBR Green Master Mix (Bio-Rad, 1708882), and gene expression was quantified using the ddCt method. The gene expression was normalized to RPL13a and B2M (see Supplemental Tables 2 and 3).

Mouse experiments. The mice were housed at the LSU Health Sciences Center animal care facility under a standard 12-hour light/dark cycle and fed with standard rodent chow and water ad libitum. Sample size was determined for each experiment. ApoE $\mathrm{E}^{-/-}$mice on the $\mathrm{C} 57 \mathrm{BL} / 6 \mathrm{~J}$ background were purchased from the Jackson Laboratory. Mice containing alleles for global Nck1 KO $\left(\mathrm{Nck}^{-/-}\right)$and conditional Nck2 deletion $\left(\mathrm{Nck} 2^{\mathrm{fl} / \mathrm{fl}}\right)$ were a gift from Tony Pawson (50), and mice containing the vascular endothelial (VE) cadherin-driven tamoxifen-inducible Cre (VE-Cadherin CreERT2) (51) were provided by Luisa Iruela-Arispe (UCLA, Los Angeles, California, USA). Mice were crossed with ApoE $\mathrm{E}^{-/-}$mice to produce iEC-Control mice (ApoE ${ }^{-/-}, \mathrm{VE}$-cadherin CreERT2 ${ }^{\mathrm{tg} / ?}$ ), Nck1-KO mice (ApoE ${ }^{-/-}, \mathrm{VE}-$ cadherin CreERT2 ${ }^{\text {tg/? }}$, Nck1 ${ }^{-/-}$), iECNck2-KO mice (ApoE-/-, VE-cadherin CreERT2 $\left.{ }^{\mathrm{tg} / \text { ? }}, \mathrm{Nck}^{\mathrm{fl} / \mathrm{fl}}\right)$, and iEC-Nck1/2-DKO mice (ApoE-/-, VE-cadherin CreERT2 ${ }^{\mathrm{tg} / \mathrm{?}}, \mathrm{Nck} 2^{\mathrm{fl} / \mathrm{fl}}$, $\left.\mathrm{Nck1}^{-/}\right)$. In humans, males show a higher predisposition to atherosclerosis compared with premenopausal females (52). However, female ApoE-KO mice show higher levels of plaque formation, suggesting that these mouse models poorly model the sexual dimorphisms observed in humans (52). Because previous studies using the PAK-Nck blocking peptide were performed in male mice (15), we focused the current study on male mice. Male mice at 8-10 weeks old were intraperitoneally injected with tamoxifen (1 mg/ $\mathrm{kg}$, MilliporeSigma) for 5 consecutive days to induce Cre nuclear translocation and gene excision. After a 2-week recovery, the 4 groups of animals were either subjected to PCL surgery as we previously reported (26) or fed HFD (TD 88137, Harlan-Teklad) for 12 weeks to induce spontaneous atherosclerotic plaque formation. For PCL, after induction of anesthesia ( $4 \%[\mathrm{v} / \mathrm{v}]$ isoflurane $\left./ \mathrm{O}_{2}\right)$, the left external carotid (below the superior thyroid artery), internal carotid, and occipital arteries were ligated with 6-0 sutures, whereas the right carotid artery was left unligated and served as an internal control. Disturbed flow within the left common carotid artery below the ligation was confirmed using Doppler ultrasound (VisualSonics VEVO3100 System), as previously described (27). Mice were euthanized either 48 hours after ligation surgery for TRIzol flush and intimal/medial and adventitial mRNA isolation or 7 days after ligation surgery for immunohistochemistry.

For bone marrow transplant experiments, Nck1-WT or Nck1-KO mice at 8-10 weeks were irradiated with 2 cycles of irradiation $(5.25$ Gy each cycle) and then received $2 \times 10^{6}$ bone marrow cells by retroorbital injection from donor mice (Nck1 WT or Nck1 KO). The animals were maintained on $0.2 \%(\mathrm{w} / \mathrm{v})$ neomycin sulfate (MilliporeSigma) in their water for 2 weeks and at 4 weeks after radiation; 4 groups of mice received HFD for an additional 12 weeks.

For atherosclerosis studies, mice were fed HFD and their body weights were monitored weekly. After the duration of the study (12 weeks), mice were euthanized by pneumothorax under anesthesia (isoflurane $/ \mathrm{O}_{2} 4 \%[\mathrm{v} / \mathrm{v}]$ ) and blood was collected by inferior vena cava puncture into heparinized collecting tubes. Plasma was collected following centrifugation at $500 \mathrm{~g}$ for 5 minutes. Tissues were collected and stored in $10 \%(\mathrm{v} / \mathrm{v})$ formalin until analysis.

Lipid analysis. Plasma total cholesterol, high density lipoprotein, and triglycerides were analyzed using commercially provided kits, as previously described (42).

Assessment of atherosclerotic lesions. The extent of atherosclerosis was assessed in the whole aorta by an en face method $(45,53)$. Lesion areas were analyzed using NIS Elements software (Nikon) and quantified as percentage of the total surface area. A second assessment of atherosclerosis was conducted in serial cross-sectional aortic roots, right carotid sinus, left carotid sinus, and brachiocephalic arteries, as described previously and according to the AHA recommendation (45, 52). Within each staining regimen, atherosclerotic sections were taken from multiple sites equidistant from an anatomical landmark (initiation of aortic valve leaflets, brachiocephalic branching point from aortic arch, or carotid bifurcation).

Immunostaining. For immunofluorescent immunocytochemistry (IF-C), cells were fixed in $4 \%(\mathrm{v} / \mathrm{v})$ formaldehyde/PBS for 10 minutes and then permeabilized with $0.1 \%(\mathrm{v} / \mathrm{v})$ Triton X-100 for 5 minutes. After blocking (10\% [v/v] horse serum/1\% [v/v] BSA/PBS for 1 hour), the cells were stained with corresponding primary antibody (Supplemental Table 1, IF-C). For nuclear p65 quantification, at least 100 cells were counted in at least 3 random fields per experimental condition from 4 independent experiments. For immunohistochemistry (IHCIF), tissue was fixed with $10 \%(\mathrm{v} / \mathrm{v})$ formalin, embedded in paraffin, and sectioned into $5-\mu \mathrm{m}$ sections as we previously published (45). Heat-mediated antigen retrieval pretreatment using $10 \mathrm{mM}$ sodium citrate buffer (Vector Biolabs) was used. All primary antibodies (Supplemental Table 1 , IHC-IF) were incubated at $4^{\circ} \mathrm{C}$ overnight. Images were captured with a Nikon microscope and analyzed using NIS Elements software. Analysis of positively stained areas was done within each atherosclerotic lesion. Areas outside the internal elastic lamina were excluded from quantification, with the exception of assessing adventitial macrophage area.

The investigators were blinded to the animal groups during the process of data collection and analysis.

Plasma cytokine analysis. Freshly isolated plasma was rapidly collected as previously published (42) and cytokine levels were assessed using LEGENDplex (BioLegend, catalog 740446) according the manufacturer's recommendations. Data analysis was performed using LEGENDplex v8.0 software.

Statistics. Data are presented as mean \pm SEM and were analyzed using GraphPad prism software (version 8.4). Data were first tested for normality using the Shapiro-Wilk test. Data showing a normal distribution were assessed for significance using the following multiple-comparisons tests: 1-way ANOVA followed by Tukey's post hoc test or 2-way ANOVA and Bonferroni's post hoc test. Data that did not show a normal distribution were analyzed using the nonparametric Kruskal-Wallis test. Statistical significance was achieved when $P$ was less than 0.05 . 
Study approval. All animal work was performed according to the National Research Council's Guide for the Care and Use of Laboratory Animals and was approved by the LSU Health Shreveport Institutional Animal Care and Use Committee. Studies involving the use of human atherosclerosis autopsy specimens were approved by the institutional review board of LSU Health Shreveport.

\section{Author contributions}

MA performed experiments, data collection, interpretation of data and analysis, and cowrote the manuscript. DW, CHA, and JGT performed atherosclerosis experiment tissue collection and analysis. AWO directed the project, interpreted results, and cowrote the manuscript.

\section{Acknowledgments}

This work was supported by a Malcolm Feist Postdoctoral Fellowship and an American Heart Association Postdoctoral Fellowship (AHA grant number 20POST35120288) to MA and by NIH grants (HL098435, HL133497, HL141155, and GM121307) to AWO. The authors acknowledge the COBRE Center for Redox Biology and Cardiovascular Disease and the Redox Molecular Signaling Core (P20GM121307) for site-directed mutagenesis of Nck1 constructs.

Address correspondence to: A. Wayne Orr, Department of Pathology, 1501 Kings Hwy, Biomedical Research Institute, Room 6-21, LSU Health Sciences Center - Shreveport, Shreveport, Louisiana 71130, USA. Phone: 318.675.5462; Email: aorr@lsuhsc.edu.
1. Libby P, Ridker PM, Hansson GK, Leducq Transatlantic Network on Atherothrombosis. Inflammation in atherosclerosis: from pathophysiology to practice. J Am Coll Cardiol. 2009;54(23):2129-2138.

2. Dai GH, et al. Distinct endothelial phenotypes evoked by arterial waveforms derived from atherosclerosis-susceptible and -resistant regions of human vasculature. Proc Natl Acad Sci U S A. 2004;101(41):14871-14876.

3. Davies PF. Flow-mediated endothelial mechanotransduction. Physiol Rev. 1995;75(3):519-560.

4. Ross R. Atherosclerosis--an inflammatory disease. N Engl JMed.1999;340(2):115-126.

5. Brooks AR, Lelkes PI, Rubanyi GM. Gene expression profiling of human aortic endothelial cells exposed to disturbed flow and steady laminar flow. Physiol Genomics. 2002;9(1):27-41.

6. Tabas I, García-Cardeña G, Owens GK. Recent insights into the cellular biology of atherosclerosis. J Cell Biol. 2015;209(1):13-22.

7. Bennett MR, Sinha S., Owens GK. Vascular smooth muscle cells in atherosclerosis. Circ Res. 2016;118(4):692-702.

8. Ridker PM, et al. Modulation of the interleukin-6 signalling pathway and incidence rates of atherosclerotic events and all-cause mortality: analyses from the Canakinumab Anti-Inflammatory Thrombosis Outcomes Study (CANTOS). Eur Heart J. 2018;39(38):3499-3507.

9. Bladt F, et al. The murine Nck SH2/SH3 adaptors are important for the development of mesodermderived embryonic structures and for regulating the cellular actin network. Mol Cell Biol. 2003;23(13):4586-4597.

10. McCarty JH. The Nck SH2/SH3 adaptor protein: a regulator of multiple intracellular signal transduction events. Bioessays. 1998;20(11):913-921.

11. Pawson T. Protein modules and signalling networks. Nature. 1995;373(6515):573-580.

12. Dubrac A, et al. NCK-dependent pericyte migration promotes pathological neovascularization in ischemic retinopathy. Nat Commun. 2018;9(1):3463.

13. Chen M, She H, Kim A, Woodley DT, Li W. Nckbeta adapter regulates actin polymerization in NIH 3T3 fibroblasts in response to platelet-derived growth factor bb. Mol Cell Biol. 2000;20(21):7867-7880.

14. Ngoenkam J, et al. Non-overlapping functions of
Nck1 and Nck2 adaptor proteins in T cell activation. Cell Commun Signal. 2014;12:21.

15. Chen J, et al. Recruitment of the adaptor protein Nck to PECAM-1 couples oxidative stress to canonical NF- $\mathrm{BB}$ signaling and inflammation. $\mathrm{Sci}$ Signal. 2015;8(365):ra20.

16. Reutershan J, et al. Blocking p21-activated kinase reduces lipopolysaccharide-induced acute lung injury by preventing polymorphonuclear leukocyte infiltration. Am J Respir Crit Care Med. 2007;175(10):1027-1035.

17. Alshahid M, et al. New susceptibility locus for obesity and dyslipidaemia on chromosome 3q22.3. Hum Genomics. 2013;7:15.

18. van der Harst P, Verweij N. Identification of 64 novel genetic loci provides an expanded view on the genetic architecture of coronary artery disease. Circ Res. 2018;122(3):433-443.

19. Khan S, et al. EndoDB: a database of endothelial cell transcriptomics data. Nucleic Acids Res. 2019;47(D1):D736-D744.

20. Hahn C, Schwartz MA. Mechanotransduction in vascular physiology and atherogenesis. Nat Rev Mol Cell Biol. 2009;10(1):53-62.

21. Walpola PL, Gotlieb AI, Cybulsky MI, Langille BL. Expression of ICAM- 1 and VCAM- 1 and monocyte adherence in arteries exposed to altered shear stress. Arterioscler Thromb Vasc Biol. 1995;15(1):2-10.

22. Clouthier DL, Harris CN, Harris RA, Martin CE, Puri MC, Jones N. Requisite role for Nck adaptors in cardiovascular development, endothelial-to-mesenchymal transition, and directed cell migration. Mol Cell Biol. 2015;35(9):1573-1587.

23. Lettau M, Pieper J, Janssen O. Nck adapter proteins: functional versatility in T cells. Cell Commun Signal. 2009;7:1.

24. Buvall L, et al. Proteasomal degradation of Nck1 but not Nck2 regulates RhoA activation and actin dynamics. Nat Commun. 2013;4:2863.

25. Jacquet K, Banerjee SL, Chartier FJM, Elowe S, Bisson N. Proteomic analysis of NCK1/2 adaptors uncovers paralog-specific interactions that reveal a new role for NCK2 in cell abscission during cytokinesis. Mol Cell Proteomics. 2018;17(10):1979-1990.

26. Yuan S, et al. Cystathionine $\gamma$-lyase modulates flow-dependent vascular remodeling. Arterioscler Thromb Vasc Biol. 2018;38(9):2126-2136.

27. Nam D, et al. Partial carotid ligation is a model of acutely induced disturbed flow, leading to rapid endothelial dysfunction and atherosclerosis. Am J Physiol Heart Circ Physiol. 2009;297(4):H1535-H1543.

28. Radu M, Chernoff J. An in vivo assay to test blood vessel permeability. J Vis Exp. 2013;(73):e50062.

29. Gimbrone MA, Topper JN, Nagel T, Anderson KR, Garcia-Cardeña G. Endothelial dysfunction, hemodynamic forces, and atherogenesis. Ann N Y Acad Sci. 2000;902:230-9; discussion 239.

30. Nakashima Y, Plump AS, Raines EW, Breslow JL, Ross R. ApoE-deficient mice develop lesions of all phases of atherosclerosis throughout the arterial tree. Arterioscler Thromb. 1994;14(1):133-140.

31. New LA, Keyvani Chahi A, Jones N. Direct regulation of nephrin tyrosine phosphorylation by Nck adaptor proteins. J Biol Chem. 2013;288(3):1500-1510.

32. Cao Z, Henzel WJ, Gao X. IRAK: a kinase associated with the interleukin-1 receptor. Science. 1996;271(5252):1128-1131.

33. Birge RB, Knudsen BS, Besser D, Hanafusa H. SH2 and SH3-containing adaptor proteins: redundant or independent mediators of intracellular signal transduction. Genes Cells. 1996;1(7):595-613.

34. Dubrac A, et al. Targeting NCK-mediated endothelial cell front-rear polarity inhibits neovascularization. Circulation. 2016;133(4):409-421.

35. Guan S, Fan J, Han A, Chen M, Woodley DT, Li W. Non-compensating roles between Nckalpha and Nckbeta in PDGF-BB signaling to promote human dermal fibroblast migration. J Invest Dermatol. 2009;129(8):1909-1920.

36. Frese $S$, et al. The phosphotyrosine peptide binding specificity of Nck1 and Nck2 Src homology 2 domains. J Biol Chem. 2006;281(26):18236-18245.

37. Huang YS, Misior A, Li LW. Novel role and regulation of the interleukin-1 receptor associated kinase (IRAK) family proteins. Cell Mol Immunol. 2005;2(1):36-39.

38. Gottipati S, Rao NL, Fung-Leung WP. IRAK1: a critical signaling mediator of innate immunity. Cell Signal. 2008;20(2):269-276.

39. Vollmer S, et al. The mechanism of activation of IRAK1 and IRAK 4 by interleukin-1 and Toll-like receptor agonists. Biochem J. 2017;474(12):2027-2038.

40. Sun $X$, et al. Activation of integrin $\alpha 5$ mediated by 
flow requires its translocation to membrane lipid rafts in vascular endothelial cells. Proc Natl Acad Sci U S A. 2016;113(3):769-774.

41. Qu D, et al. Focal TLR4 activation mediates disturbed flow-induced endothelial inflammation. Cardiovasc Res. 2020;116(1):226-236.

42. Al-Yafeai Z, Yurdagul A, Peretik JM, Alfaidi M, Murphy PA, Orr AW. Endothelial FN (fibronectin) deposition by $\alpha 5 \beta 1$ integrins drives atherogenic inflammation. Arterioscler Thromb Vasc Biol. 2018;38(11):2601-2614.

43. Kiosses WB, et al. A dominant-negative p65 PAK peptide inhibits angiogenesis. Circ Res. 2002;90(6):697-702.

44. Orr AW, et al. Matrix-specific p21-activated kinase activation regulates vascular permeability in atherogenesis. J Cell Biol. 2007;176(5):719-727.

45. Chen J, Green J, Yurdagul A, Albert P, McInnis $\mathrm{MC}$, Orr AW. $\alpha \mathrm{v} \beta 3$ Integrins mediate flow- induced NF-кB activation, proinflammatory gene expression, and early atherogenic inflammation. Am J Pathol. 2015;185(9):2575-2589.

46. Yuan S, Pardue S, Shen X, Alexander JS, Orr AW, Kevil CG. Hydrogen sulfide metabolism regulates endothelial solute barrier function. Redox Biol. 2016;9:157-166.

47. Dull T, et al. A third-generation lentivirus vector with a conditional packaging system.J Virol. 1998;72(11):8463-8471.

48. Orr AW, Sanders JM, Bevard M, Coleman E, Sarembock IJ, Schwartz MA. The subendothelial extracellular matrix modulates NF-kappaB activation by flow: a potential role in atherosclerosis. JCell Biol. 2005;169(1):191-202.

49. Orr AW, Hahn C, Blackman BR, Schwartz MA. p21-activated kinase signaling regulates oxidant-dependent NF-kappa B activation by flow. Circ Res. 2008;103(6):671-679.
50. Fawcett JP, et al. Nck adaptor proteins control the organization of neuronal circuits important for walking. Proc Natl Acad Sci U S A. 2007;104(52):20973-20978.

51. Monvoisin A, Alva JA, Hofmann JJ, Zovein AC, Lane TF, Iruela-Arispe ML. VE-cadherinCreERT2 transgenic mouse: a model for inducible recombination in the endothelium. Dev Dyn. 2006;235(12):3413-3422.

52. Daugherty A, et al. Recommendation on design, execution, and reporting of animal atherosclerosis studies: a scientific statement from the American Heart Association. Arterioscler Thromb Vasc Biol. 2017;37(9):e131-e157.

53. Alfaidi MA, et al. Dietary docosahexaenoic acid reduces oscillatory wall shear stress, atherosclerosis, and hypertension, most likely mediated via an IL-1-mediated mechanism. JAm Heart Assoc. 2018;7(13):e008757. 